\title{
4-二甲胺基吡啶-磁自由基促进的缺电子烯烃区域选择性磞氢化反应
}

\author{
黄云帅靳小慧张风莲* 汪义丰* \\ (中国科学技术大学化学系 合肥 230026)
}

\begin{abstract}
摘要 含嗍有机化合物在合成化学中具有重要的应用价值, 嗍氢化反应是构建有机嗍化合物最常用的方法之一. 利用 4-二甲氨基吡啶嗍自由基与缺电子烯烃的自由基硼氢化反应，高区域选择性地构建了 $\alpha$-硼取代产物. 该反应条件温和, 官能团容忍性好, 底物范围广. 多种 $\alpha, \beta$-不饱和酯、酰胺、羧酸、腈、三氟甲基化合物、砜以及磷酸酯均能顺利发生反 应，得到的含嗍产物可以进一步应用于后续碳-碳键的构建. 关键词 4-二甲氨基吡啶硼自由基; 缺电子烯烃; 区域选择性；硼氢化反应；自由基化学
\end{abstract}

\section{4-Dimethylaminopyridine-Boryl Radical Promoted Regioselective Radical Hydroboration of Electron-Deficient Alkenes}

\author{
Huang, Yunshuai Jin, Xiaohui Zhang, Fenglian* Wang, Yifeng* \\ (Department of Chemistry, University of Science and Technology of China, Hefei 230026)
}

\begin{abstract}
Organoboron compounds have shown significant applications in modern chemical synthesis. Hydroboration of alkenes is among the most widely used methods to access these targets. Herein, a regioselective radical hydroboration reaction of electron-deficient alkenes with 4-dimethylaminopyridine (DMAP)-boryl radical for the synthesis of $\alpha$-boryl functionalized molecules is reported. The reaction features specific $\alpha$-regioselectivity, mild reaction conditions, good functional group tolerance, and broad substrate scope. $\alpha, \beta$-Unsaturated esters, amides, carboxylic acid, nitrile, trifluoromethyl molecule, sulfone, and phosphonate are viable substrates for this reaction. The resulting $\alpha$-boryl functionalized molecules can be further transformed to various useful building blocks.
\end{abstract}

Keywords 4-dimethylaminopyridine-boryl radical; electron-deficient alkenes; regioselectivity; hydroboration; radical chemistry

\section{Introduction}

Organoboron compounds have been widely used in synthetic chemistry, medicinal chemistry, and material sciences. ${ }^{[1]}$ Hydroboration of alkenes is among the most important method to prepare organoboron molecules. ${ }^{[2]}$ Although a large number of reactions with electron-deficient alkenes have been reported for the synthesis of $\beta$-borylated products, ${ }^{[3]}$ the methods to access $\alpha$-borylated products have been less developed. This is because that these products are usually unstable and can undergo facile 1,3-boron shift to give thermodynamically more stable $O$-boron enolates. ${ }^{[4]}$ To address this issue, the presence of Lewis base moiety that can quaternize the boron center is crucial, so that the 1,3-boron shift can be suppressed and the products become moisture stable. ${ }^{[5]}$ In 1977, Sucrow et $a l .{ }^{[6]}$ described a hydroboration of electron-poor alkenes bearing an intramolecular hydrazine moiety, where the imino nitrogen atom acted as donor to stabilize the borane center. Recently, Chiu et al..$^{[7]}$ disclosed an elegant method to access $\alpha$-boryl esters and amides through copper-catalyzed hydroboration of $\alpha, \beta$-unsaturated carbonyl compounds. Although the presence of an intramolecular coordinating group was not requisite, the addition of a phosphine ligand was vital for the formation of C-boron enolates. Very recently, the group of Ingleson et al. ${ }^{[8]}$ reported an iodine-mediated hydroboration of $\alpha, \beta$-unsaturated esters using $N$-heterocyclic carben ( $\mathrm{NHC}$ )- $\mathrm{BH}_{3}$, whereas the substrate scope and generality were limited.

In recent years, Lewis-base boryl radicals have shown

\footnotetext{
* Corresponding authors. E-mail: zf19@ustc.edu.cn; yfwangzj@ustc.edu.cn

Received March 23, 2021; revised April 9, 2021; published online April 25, 2021.

Dedicated to Professor Ilhyong Ryu on the occasion of his 70th birthday.

Project supported by the National Natural Science Foundation of China (No. 21971226) and the Fundamental Research Funds for the Central Universities (No. WK2060000017).

国家自然科学基金(No. 21971226)和中央高校基本科研业务费专项资金(No. WK2060000017)资助项目
} 
robust synthetic applications, ${ }^{[9]}$ in particular for the synthesis of organoboron molecules ${ }^{[10]}$ that are difficult to access by traditional ionic and transition metal-catalyzed methods. Our group has recently reported a radical hydroboration of electron-deficient alkenes using $\mathrm{NHC}-\mathrm{BH}_{3}$ as the boryl radical precursor (Scheme 1). ${ }^{[11]}$ The reaction features exclusive $\alpha$-regioselectivity and the resulting $\alpha$-boryl carbonyl products are amenable for various transformations. However, the reactions still need to be conducted under higher reaction temperature $\left(60 \sim 95{ }^{\circ} \mathrm{C}\right)$ in order to promote the initiation of the radical initiator, such as diazo compounds and peroxides. Moreover, the preparation of $\mathrm{NHC}-\mathrm{BH}_{3}$ requires a two-step reaction, wherein a strong base is required to enable the formation of NHC in situ. ${ }^{[12]}$ We envisaged that if a more readily accessible boryl radical precursor could be also used in analogous hydroboration, meanwhile the initiation could be realized at room temperature, a more practical and economical protocol would be accomplished. 4-Dimethylaminopyridine (DM$\mathrm{AP})-\mathrm{BH}_{3}$ is readily prepared by mixing DMAP and $\mathrm{BH}_{3}$ THF in tetrahydrofuran (THF) followed by simple filtration. More importantly, its $\mathrm{B}-\mathrm{H}$ bond has a relatively low bond dissociation energy $(338.9 \mathrm{~kJ} / \mathrm{mol})$ and can be easily cleaved to generate DMAP- $\mathrm{BH}_{2} \bullet$ in the presence of a radical initiator. ${ }^{[13]}$ Very recently, we disclosed that this radical is able to promote sequential defluorination of trifluoroacetamides and trifluoroacetates to selectively form di- and mono-fluorinated molecules. ${ }^{[14]}$ Herein, we describe a mild radical hydroboration of electron-deficient alkenes using DMAP-BH $H_{3}$ as the boryl radical precursor and $\mathrm{Et}_{3} \mathrm{~B} / \mathrm{O}_{2}$ as radical initiator. The radical reaction is carried out at room temperature and tolerates a broad range of functional groups.

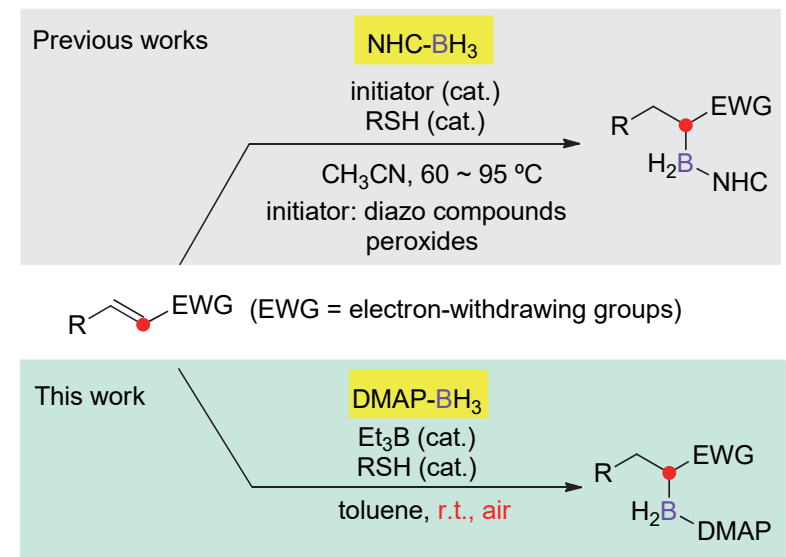

Scheme 1 Radical hydroboration of electron-deficient alkenes

\section{Results and discussion}

We commenced our study by examining the reaction of ethyl cinnamate (1a) and $\mathrm{DMAP}-\mathrm{BH}_{3}$ in the presence of a radical initiator. To our delight, the reaction proceeded smoothly using 2,2-azobis(isobutyronitrile) (AIBN) as the radical initiator and $\mathrm{PhSH}$ as the polarity reversal catalyst, ${ }^{[15]}$ affording $\alpha$-boryl ester $3 \mathbf{a a}$ in $46 \%$ yield (Table 1 ,
Entry 1). Switching the radical initiator to 1,1'-azobis(cyclohexanecarbonitrile) (ACCN), 2,2'-azobis(2,4-dimethyl)valeronitrile $(\mathrm{ABVN})$, and di-tert-butyl hyponitrite (TBHN) led to increased yields (Entries 2 4). However, heating was always required to initiate the reaction. Notably, when $\mathrm{Et}_{3} \mathrm{~B}$ /air was utilized as the radical initiator and the reaction conducted at room temperate, the reaction proceeded nicely as well, furnishing the desired product in $85 \%$ yield (Entry 5 ). Reducing the amount of $\mathrm{PhSH}$ to 20 mol\% could maintain high efficiency and 3aa was isolated in $84 \%$ yield (Entry 6 ). It should be noted that no reaction occurred in the absence of $\mathrm{Et}_{3} \mathrm{~B}$, implying a radical reaction mechanism where $\mathrm{Et}_{3} \mathrm{~B}$ reacts with $\mathrm{O}_{2}$ to generate ethyl radical that initiate the ensuing radical chain process (Entry 7). $\mathrm{NH}_{3}-\mathrm{BH}_{3}$ is another class of broadly applicable complex that may also serve as the boryl radical precursor. ${ }^{[16]}$ However, no reaction was detected under the same reaction conditions (Entry 8 ).

Table 1 Optimization of reaction conditions ${ }^{a}$

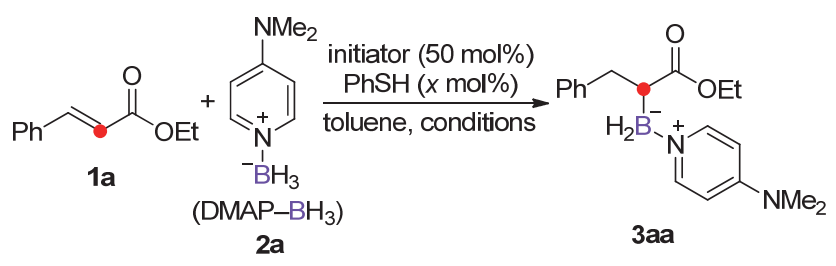

\begin{tabular}{ccccc}
\hline Entry & PhSH $(x$ mol\%) & Initiator & Conditions & $\begin{array}{c}\text { Yield }{ }^{b} \% \text { of } \\
\text { 3aa }\end{array}$ \\
\hline 1 & 50 & AIBN & $80{ }^{\circ} \mathrm{C}, 9 \mathrm{~h}$ & 46 \\
2 & 50 & $\mathrm{ACCN}$ & $95{ }^{\circ} \mathrm{C}, 9 \mathrm{~h}$ & 55 \\
3 & 50 & $\mathrm{ABVN}$ & $60{ }^{\circ} \mathrm{C}, 4 \mathrm{~h}$ & 69 \\
4 & 50 & $\mathrm{TBHN}$ & $60{ }^{\circ} \mathrm{C}, 4 \mathrm{~h}$ & 83 \\
5 & 50 & $\mathrm{Et}$ B & Air, r.t., $9 \mathrm{~h}$ & $85^{c}$ \\
6 & 20 & $\mathrm{Et}_{3} \mathrm{~B}$ & Air, r.t., $9 \mathrm{~h}$ & $84^{c}$ \\
7 & 20 & - & $\mathrm{O}_{2}$, r.t., $9 \mathrm{~h}$ & $0(>95)^{d}$ \\
$8^{e}$ & 20 & $\mathrm{Et}_{3} \mathrm{~B}$ & Air, r.t., $9 \mathrm{~h}$ & $0(>95)^{d}$ \\
\hline
\end{tabular}

${ }^{a}$ Unless otherwise noticed, the reactions were carried out on a $0.4 \sim 0.5 \mathrm{mmol}$ scale of $1 \mathbf{a}$ with 1.2 equiv. of $\mathbf{2 a}$ in the presence of radical initiator $(50 \mathrm{~mol} \%)$ and $\mathrm{PhSH}(x \mathrm{~mol} \%)$ in toluene $(0.1 \mathrm{~mol} / \mathrm{L}) ;{ }^{b} \mathrm{NMR}$ yield using tetrachloroethane as an internal standard; ${ }^{c}$ Isolated yield; ${ }^{d}$ Recovery yield of $1 \mathrm{a}$ is shown in parentheses; ${ }^{e} \mathrm{NH}_{3}-\mathrm{BH}_{3}$ was used instead of DMAP-BH

Using the optimized reaction conditions, the substrate scope of this radical hydroboration of electron-deficient alkenes for the synthesis of $\alpha$-borylated products was examined (Table 2). The reaction shows excellent functional group capability. Ethyl cinnamates bearing various substituents, regardless of their electronic nature or substitution pattern, performed well under the standard conditions to furnish a series of substituted $\alpha$-boryl- $\beta$-aryl esters (3ba $\sim 3 \mathbf{j a})$ in moderate to good yields. The phenyl ring could be replaced with naphthalene (3ka), indole (3la), or furan (3ma). A gram scale synthesis of 3ka was achieved in $64 \%$ yield. Radical hydroboration of tert-butyl and 2-naphthyl cinnamate afforded the corresponding $\alpha$ borylated products (3na and 3oa) efficiently. Nature product cumarin was transformed into the hydroborated 
Table 2 Scope of radical hydroboration of $\alpha, \beta$-unsaturated carbonyl compounds ${ }^{a}$

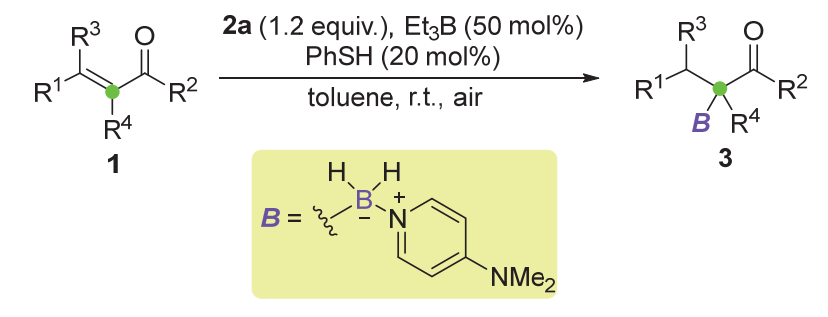

$\alpha$-boryl- $\beta$-aryl esters

3ba, $\mathrm{R}=2-\mathrm{Me}, 70 \%$

3ca, $\mathrm{R}=2-\mathrm{Cl}, 72 \%$

3da, $R=2-\mathrm{CN}, 61 \%$

3ea, $R=3-\mathrm{Me}, 68 \%$

3fa, $\mathrm{R}=3-\mathrm{OH}, 51 \%$

3ga, $\mathrm{R}=4-\mathrm{Ph}, 69 \%$

3ha, $R=4-F, 73 \%^{b}$

3ia, $R=4-C N, 43 \%$

3ja, $\mathrm{R}=4-\mathrm{NMe}_{2}, 49 \%$<smiles>[B]C(Cc1ccco1)C(=O)OCC</smiles>

3ma, $57 \%$<smiles>[B]C1Cc2ccccc2OC1=O</smiles>

3 pa, $65 \%^{b}$<smiles>[B]C(CC)(Cc1ccccc1)CC(C)(C)C(=O)OCC</smiles>

3sa, $64 \%^{b}$<smiles>[B]C(Cc1ccccc1)C(=O)OCCC</smiles>

3na, $82 \%$<smiles>[B]C(Cc1ccccc1)C(=O)OC/C=C/c1ccccc1</smiles>

$3 q a, 39 \%^{b}$<smiles>CCOC(=O)C(C(=O)c1ccccc1)[N+](=O)[O-]</smiles>

3sa', $5 \%^{b}(d r=12.8: 1)$

$\alpha$-boryl- $\beta$-aryl amides and acids<smiles>C=CCN(CC=C)C(=O)C(Br)Cc1ccccc1</smiles><smiles>[B]C(Cc1ccc(OCC=C)cc1)C(=O)N1CCOCC1</smiles>

3va, $62 \%$<smiles>[B]C(Cc1ccccc1)C(=O)N1CCCC1=O</smiles>

$3 \times a, 22 \%^{b}$

3ya, $76 \%$<smiles>C#CCN(C)C(=O)C(Br)(Cc1ccccc1)C(C)(C)C</smiles><smiles>[B]C(Cc1ccccc1)C(=O)N(Cc1ccccc1)Cc1ccccc1</smiles>

3wa, $65 \%^{b}$<smiles>O=C(O)C(Br)Cc1ccccc1</smiles>

$\alpha$-boryl- $\beta$-alkyl esters<smiles>[B]B(C(=O)OCC)C(C)C</smiles>

3za, $17 \%$

${ }^{a}$ Unless otherwise noticed, the reactions were carried out on a $0.4 \sim 0.5 \mathrm{mmol}$ scale of $\mathbf{1}$ with 1.2 equiv. of $\mathbf{2 a}$ in the presence of $\mathrm{Et}_{3} \mathrm{~B}(50 \mathrm{~mol} \%)$ and $\mathrm{PhSH}$ $(20 \mathrm{~mol} \%)$ in toluene $(0.1 \mathrm{~mol} / \mathrm{L})$ under air; ${ }^{b} \mathrm{PhSH}(50 \mathrm{~mol} \%)$ was used; ${ }^{c} \mathrm{~A}$ gram scale synthesis $(1.06 \mathrm{~g}, 4.68 \mathrm{mmol})$.

product in $65 \%$ yield (3pa). The reaction of 1,6-diene 1q delivered 3qa as the sole product, without the detection of any intramolecular cyclization product, presumably because hydrogen atom abstraction of the generated carbon radical is much faster than cyclization process. Increasing the steric congestion at the $\beta$-carbon resulted in a slight decrease of the reaction efficiency (3ra). Notably, introducing a methyl group at the $\alpha$-carbon did not retard the $\alpha$-addition, giving $\alpha$-borylated product 3sa in $64 \%$ yield along with $5 \%$ yield of $\beta$-borylated product 3sa'. It is worth noting that the same substrate gave a reverse regioselectivity $\left(3 \mathbf{s a}: 3 \mathbf{s a}^{\prime}=1: 2.4\right)$ when the reaction was carried out using $\mathrm{NHC}-\mathrm{BH}_{3}$ as the boryl radical precursor and AIBN as the radical initiator at $80{ }^{\circ} \mathrm{C} .{ }^{[11 a]}$ This indicates that both the Lewis base moiety of the boryl radical and the reaction temperature are crucial for regioselectivity control. Radical hydroboration of other types of $\alpha, \beta$-unsaturated carbonyl compounds was also studied. An array of $\alpha$-borylated amides (3ta $\sim 3 \mathbf{x a})$ were successfully constructed. Both terminal alkene (3ta) and alkyne (3ua) moieties were tolerated. Cinnamic acid $\mathbf{1 y}$ was also amenable and $\alpha$-borylated acid 3ya was isolated in $76 \%$ yield. When the $\beta$-aryl group was switched to an alkyl one, the reaction became sluggish, affording 3za in a low yield. Such an unfavorable reaction is probably due to the less stabilization of this alkyl group to the resulting radical addition intermediate.

This radical hydroboration protocol could be further applied to various electron-deficient alkenes. As shown in Table 3, electron-deficient alkenes bearing different substituents, such as $\mathrm{CN}, \mathrm{CF}_{3}$ and $\mathrm{SO}_{2} \mathrm{Ph}$, were converted into the corresponding $\alpha$-borylated products with good efficiency and $\alpha$-regioselectivity. However, the reaction of $\mathbf{4 d}$ produced $\beta$-borylated $\mathbf{5} \mathbf{d}^{\prime}$ as the major product along with

Table 3 Scope of radical $\alpha$-borylation of various electron-deficient alkenes $^{a}$

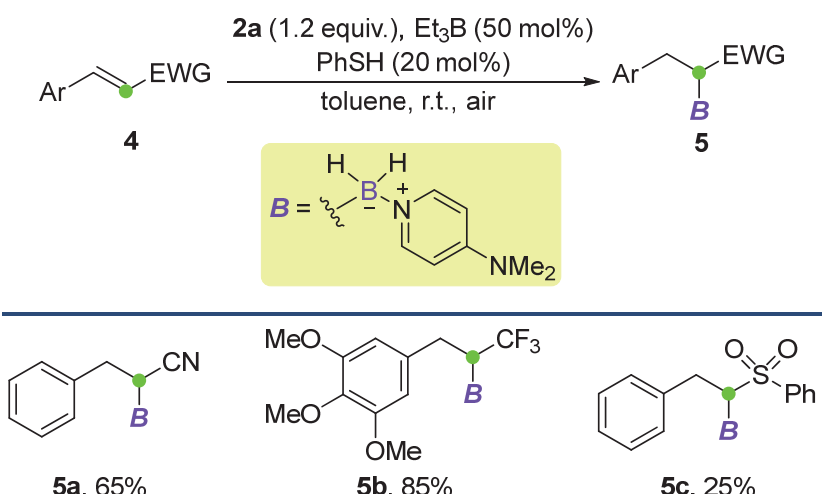

5 a, $65 \%$

5b, $85 \%$

$5 c, 25 \%$

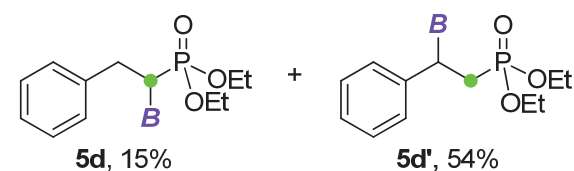

${ }^{a}$ Unless otherwise noticed, the reactions were carried out on a $0.4 \sim 0.5 \mathrm{mmol}$ scale of $\mathbf{4}$ with 1.2 equiv. of $\mathbf{2 a}$ in the presence of $\mathrm{Et}_{3} \mathrm{~B}(50 \mathrm{~mol} \%)$ and $\mathrm{PhSH}$ $(20 \mathrm{~mol} \%)$ in toluene $(0.1 \mathrm{~mol} / \mathrm{L})$ under air. 
$15 \%$ of $\alpha$-borylated product $\mathbf{5 d}$. This inverse regioselectivity is likely due to the dual effect resulted from the enhanced nucleophilicity of DMAP- $\mathrm{BH}_{2} \bullet$ and the increased steric hinderance of the phosphate moiety. It should be noted that the reactions of $\alpha, \beta$-unsaturated aldehydes and ketones could not give the desired hydroboration products, and complex mixtures containing the reduction of the carbonyl groups were observed.

Next, a radical clock experiment was performed to clarify the radical reaction mechanism. ${ }^{[17]}$ As shown in Scheme $2 \mathrm{a}$, the reaction of $\mathbf{6}$ bearing a cyclopropane ring at the $\beta$-carbon gave ring-opening product 7 under the standard reaction conditions. Based on this result as well as our previous findings, ${ }^{[11]}$ a radical chain reaction mechanism was proposed and outlined in Scheme 2b. By using $\mathrm{Et}_{3} \mathrm{~B} / \mathrm{O}_{2}$ as the initiation system, DMAP- $\mathrm{BH}_{2} \bullet$ is generated via hydrogen atom abstraction. Regioselective addition of DMAP- $\mathrm{BH}_{2} \bullet$ to the $\alpha$-carbon of electron-deficient alkenes 1 affords a carbon radical intermediate II. Hydrogen atom transfer from $\mathrm{PhSH}$ to II delivers desired $\alpha$-borylated products 3. ${ }^{[18]}$ The generated sulfur radical then abstracts a hydrogen atom from $\mathrm{DMAP}_{-} \mathrm{BH}_{3}$, regenerating $\mathrm{PhSH}$ and DMAP- $\mathrm{BH}_{2} \bullet$ to propagate the radical chain process. The rationale of $\alpha$-regioselectivity is likely the same with the reaction of $\mathrm{NHC}-\mathrm{BH}_{3}$ as demonstrated in our previous studies. ${ }^{[11]}$

(a) A radical clock experiment

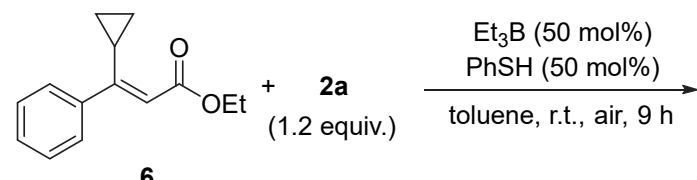

6<smiles></smiles>

(b) A proposed reaction mechanism

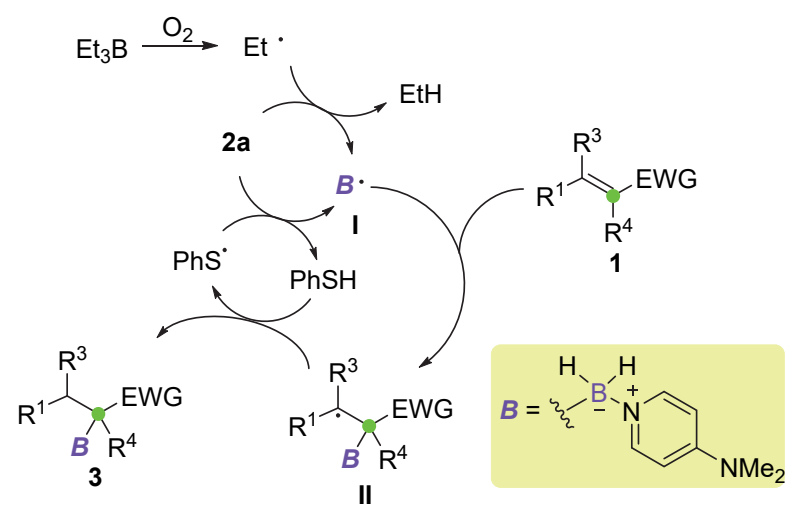

Scheme 2 Mechanistic study and a plausible mechanism

The synthetic applicability of $\alpha$-borylated products was further demonstrated (Scheme 3). Reduction of the ester group of 3ka with DIBAL-H, followed by treatment with pinacol in the presence of $N$-chloro-succinimide (NCS) and trimethylamine, and protection of the generated hydroxyl group with TBS afforded protected $\beta$-hydroxy boronate $\mathbf{8}$ in $63 \%$ overall yield. Coupling reactions of 8 with furan and 3-fluoro- pydine were realized using Aggarwal's arylation protocol, ${ }^{[19]}$ delivering arylated products $\mathbf{9}$ and $\mathbf{1 0}$ in good yields. Homologation of boronate $\mathbf{8}$ followed by further oxidation produced propanediol derivative $\mathbf{1 1}$.

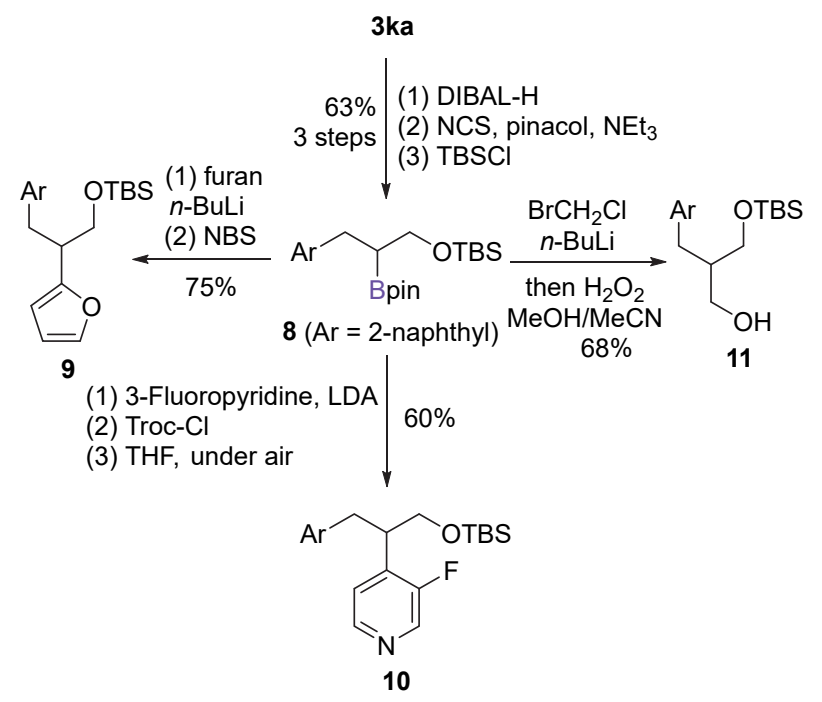

Scheme 3 Diversifications of hydroboration product 3ka

\section{Conclusions}

In summary, a regioselective radical hydroboration of electron-deficient alkenes was developed by using DMAP$\mathrm{BH}_{3}$ under $\mathrm{Et}_{3} \mathrm{~B} / \mathrm{O}_{2}$ initiation system. DMAP-BH$H_{3}$ is readily available and bench stable, and it is an ideal boryl radical precursor to make organoboron compounds. The reaction is carried out at room temperature and features specific $\alpha$-regioselectivity and broad substrate scope. This method is capable of easily constructing various $\alpha$-borylated products, such as $\alpha$-boryl esters, amides, acids, nitriles, trifluoromethyl compounds, and sulfones from simple starting materials.

\section{Experimental section}

\subsection{General experimental information}

${ }^{1} \mathrm{H}$ NMR (400 MHz) spectra were recorded on a Bruker Avance 400 spectrometer in $\mathrm{CDCl}_{3}$ [using $\mathrm{CDCl}_{3}(\delta$ 7.26), $\mathrm{CD}_{3} \mathrm{CN}\left(\delta 1.94\right.$ (quint)) or $\mathrm{C}_{6} \mathrm{D}_{6}(\delta 7.16)$ as the internal standard]. ${ }^{13} \mathrm{C}$ NMR (100 MHz) spectra on a Bruker Avance 400 spectrometer in $\mathrm{CDCl}_{3}$ [using $\mathrm{CDCl}_{3}(\delta 77.0)$, $\mathrm{CD}_{3} \mathrm{CN}(\delta 118.7)$ or $\mathrm{C}_{6} \mathrm{D}_{6}(\delta 128.06)$ as internal standard]. High-resolution mass spectra were obtained with a Water XEVO G2 Q-Tof (Waters Corporation). Melting points were uncorrected and were recorded on a Buchi B-54 melting point apparatus. Flash column chromatography was performed using Merck silica gel 60 with distilled solvents. Commercially available reagents were purchased from Energy Chemical, J \& K Scientific, Adamas-beta and 
Sigma-Aldrich Co., Inc.

Compounds 1a, 1p, 1y, and 4a were purchased and used directly without additional purification. $\mathrm{DMAP}^{-\mathrm{BH}_{3}}$ (2a) was prepared according to the literature procedure. ${ }^{[13]}$

\subsection{Regioselective radical hydroboration of electron-} deficient alkenes

General procedure: a solution of $1(0.4 \sim 0.5 \mathrm{mmol}, 1$ equiv.), 2a (1.2 equiv.), $\mathrm{Et}_{3} \mathrm{~B}$ (50 $\left.\mathrm{mol} \%\right)$, and $\mathrm{PhSH}(20$ $\mathrm{mol} \%)$ in toluene $(2.5 \mathrm{~mL})$ was stirred at room temperature for $9 \mathrm{~h}$ under air. After evaporation of solvent, the resulting crude material was purified by flash column chromatography to provide the pure products 3 .

(4-Dimethylaminopyridine)(1-ethoxy-1-oxo-3-phenylpropan-2-yl)dihydroborate (3aa): The yield is $84 \%$ (110.7 $\mathrm{mg}$ as a white solid) after column chromatography. m.p. $80 \sim 81{ }^{\circ} \mathrm{C} ;{ }^{1} \mathrm{H}$ NMR $\left(400 \mathrm{MHz}, \mathrm{CDCl}_{3}\right) \delta: 1.02(\mathrm{t}, J=7.2$ $\mathrm{Hz}, 3 \mathrm{H}), 2.22 \sim 2.29(\mathrm{~m}, 1 \mathrm{H}), 2.64(\mathrm{dd}, J=3.6,14.4 \mathrm{~Hz}$, $1 \mathrm{H}), 2.93$ (dd, $J=10.8,14.2 \mathrm{~Hz}, 1 \mathrm{H}), 3.05$ (s, 6H), 3.88 (q, $J=7.2 \mathrm{~Hz}, 2 \mathrm{H}), 6.47$ (d, $J=7.6 \mathrm{~Hz}, 2 \mathrm{H}), 7.04 \sim 7.10(\mathrm{~m}$, $1 \mathrm{H}), 7.14 \sim 7.21(\mathrm{~m}, 4 \mathrm{H}), 7.94(\mathrm{~d}, J=7.2 \mathrm{~Hz}, 2 \mathrm{H}) ;{ }^{13} \mathrm{C}$ NMR $\left(100 \mathrm{MHz}, \mathrm{CDCl}_{3}\right) \delta: 14.3,36.3,39.3,58.4,106.0$, $124.8,127.7,128.3,144.4,146.4,154.9,180.4 ;{ }^{11} \mathrm{~B}$ NMR $\left(128.4 \mathrm{MHz}, \mathrm{CDCl}_{3}\right) \delta$ : -5.3 (br s, 1B); ESI-HRMS calcd for $\mathrm{C}_{18} \mathrm{H}_{25} \mathrm{BN}_{2} \mathrm{O}_{2} \mathrm{Na}(\mathrm{M}+\mathrm{Na})^{+}$335.1907, found 335.1903 .

(4-Dimethylaminopyridine)(1-ethoxy-1-oxo-3-(o-tolyl)propan-2-yl)dihydroborate (3ba): The yield is 70\% (92.0 $\mathrm{mg}$ as a white solid) after column chromatography. m.p. 65 $67{ }^{\circ} \mathrm{C} ;{ }^{1} \mathrm{H}$ NMR $\left(400 \mathrm{MHz}, \mathrm{CDCl}_{3}\right) \delta: 1.03$ (t, $J=7.2$ $\mathrm{Hz}, 3 \mathrm{H}), 2.20 \sim 2.25(\mathrm{~m}, 1 \mathrm{H}), 2.29(\mathrm{~s}, 3 \mathrm{H}), 2.65(\mathrm{dd}, J=$ $3.6,14.8 \mathrm{~Hz}, 1 \mathrm{H}), 2.92$ (dd, $J=10.4,14.8 \mathrm{~Hz}, 1 \mathrm{H}), 3.09$ (s, $6 \mathrm{H}), 3.89(\mathrm{qd}, J=2.0,7.2 \mathrm{~Hz}, 2 \mathrm{H}), 6.50(\mathrm{~d}, J=7.6 \mathrm{~Hz}$, $2 \mathrm{H}), 7.96 \sim 7.08(\mathrm{~m}, 3 \mathrm{H}), 7.16 \sim 7.21(\mathrm{~m}, 1 \mathrm{H}), 7.99(\mathrm{~d}, J=$ $7.2 \mathrm{~Hz}, 2 \mathrm{H}) ;{ }^{13} \mathrm{C} \mathrm{NMR}\left(100 \mathrm{MHz}, \mathrm{CDCl}_{3}\right) \delta: 14.4,19.5$, 33.3, 39.4, 58.5, 106.1, 125.0, 125.3, 128.6, 129.6, 136.0, 142.5, 146.6, 155.0, 180.6; ${ }^{11} \mathrm{~B}$ NMR (128.4 MHz, $\left.\mathrm{CDCl}_{3}\right)$ $\delta$ : -4.5 (br s, 1B); ESI-HRMS calcd for $\mathrm{C}_{19} \mathrm{H}_{27} \mathrm{BN}_{2} \mathrm{O}_{2} \mathrm{Na}$ $(\mathrm{M}+\mathrm{Na})^{+}$349.2063, found 349.2060.

(3-(2-Chlorophenyl)-1-ethoxy-1-oxopropan-2-yl)(4dimethylaminopyridine)dihydroborate (3ca): The yield is $72 \%(75.3 \mathrm{mg}$ as a white solid) after column chromatography. m.p. 86 89 ${ }^{\circ} \mathrm{C}$; ${ }^{1} \mathrm{H}$ NMR $\left(400 \mathrm{MHz}, \mathrm{CDCl}_{3}\right) \delta$ : $1.05(\mathrm{t}, J=7.2 \mathrm{~Hz}, 3 \mathrm{H}), 2.30 \sim 2.37(\mathrm{~m}, 1 \mathrm{H}), 2.79(\mathrm{dd}, J=$ 3.6, $14.4 \mathrm{~Hz}, 1 \mathrm{H}), 2.98$ (dd, $J=10.8,14.4 \mathrm{~Hz}, 1 \mathrm{H}), 3.10$ (s, $6 \mathrm{H}), 3.91(\mathrm{qd}, J=0.8,7.2 \mathrm{~Hz}, 2 \mathrm{H}), 6.48 \sim 6.55(\mathrm{~m}, 2 \mathrm{H})$, $7.00 \sim 7.11(\mathrm{~m}, 2 \mathrm{H}), 7.22 \sim 7.32(\mathrm{~m}, 2 \mathrm{H}), 7.98 \sim 8.05(\mathrm{~m}$, $2 \mathrm{H}) ;{ }^{13} \mathrm{C} \mathrm{NMR}\left(100 \mathrm{MHz}, \mathrm{CDCl}_{3}\right) \delta: 14.4,34.0,39.5,58.6$, $106.1,126.2,126.4,129.0,130.6,134.0,141.7,146.7$, 155.0, 180.2; ${ }^{11} \mathrm{~B}$ NMR (128.4 MHz, $\left.\mathrm{CDCl}_{3}\right) \delta$ : -4.7 (br s, 1B); ESI-HRMS calcd for $\mathrm{C}_{18} \mathrm{H}_{24} \mathrm{BClN}_{2} \mathrm{O}_{2} \mathrm{Na}(\mathrm{M}+\mathrm{Na})^{+}$ 369.1517 , found 369.1510 .

(3-(2-Cyanophenyl)-1-ethoxy-1-oxopropan-2-yl)(4dimethylaminopyridine)dihydroborate (3da): The yield is $61 \%(61.7 \mathrm{mg}$ as a white solid) after column chromatography. m.p. $200 \sim 203{ }^{\circ} \mathrm{C}$; ${ }^{1} \mathrm{H}$ NMR $\left(400 \mathrm{MHz}, \mathrm{CDCl}_{3}\right) \delta$ : $1.04(\mathrm{t}, J=7.0 \mathrm{~Hz}, 3 \mathrm{H}), 2.35(\mathrm{~m}, 1 \mathrm{H}), 2.86(\mathrm{dd}, J=2.1$,
$14.1 \mathrm{~Hz}, 1 \mathrm{H}), 3.07 \sim 3.14(\mathrm{~m}, 7 \mathrm{H}), 3.89(\mathrm{q}, J=6.8 \mathrm{~Hz}$, $2 \mathrm{H}), 6.52(\mathrm{q}, J=6.4 \mathrm{~Hz}, 2 \mathrm{H}), 7.14 \sim 7.18(\mathrm{~m}, 1 \mathrm{H}), 7.39 \sim$ 7.40 (d, $J=3.6 \mathrm{~Hz}, 2 \mathrm{H}), 7.50$ (d, $J=7.6 \mathrm{~Hz}, 1 \mathrm{H}), 8.01$ (d, $J=6.4 \mathrm{~Hz}, 2 \mathrm{H}) ;{ }^{13} \mathrm{C}$ NMR $\left(100 \mathrm{MHz}, \mathrm{CDCl}_{3}\right) \delta: 14.4$, $34.9,39.5,58.7,106.2,112.2,118.5,125.6,129.7,132.2$, 132.4, 146.6, 148.5, 155.1, 179.7; ${ }^{11} \mathrm{~B}$ NMR (128.4 MHz, $\left.\mathrm{CDCl}_{3}\right) \delta$ : -6.4 (br s, 1B); ESIHRMS calcd for $\mathrm{C}_{19} \mathrm{H}_{24} \mathrm{~B}-$ $\mathrm{N}_{3} \mathrm{O}_{2} \mathrm{Na}(\mathrm{M}+\mathrm{Na})^{+} 360.1859$, found 360.1859.

(4-Dimethylaminopyridine)(1-ethoxy-1-oxo-3-( $m$-tolyl)propan-2-yl)dihydroborate (3ea): The yield is 68\% (67.2 $\mathrm{mg}$ as colorless liquid) after column chromatography. ${ }^{1} \mathrm{H}$ NMR (400 MHz, $\left.\mathrm{CDCl}_{3}\right) \delta: 1.04(\mathrm{t}, J=7.2 \mathrm{~Hz}, 3 \mathrm{H})$, $2.21 \sim 2.29(\mathrm{~m}, 4 \mathrm{H}), 2.61(\mathrm{dd}, J=3.6,14.4 \mathrm{~Hz}, 1 \mathrm{H}), 2.90$ (dd, $J=10.4,14.4 \mathrm{~Hz}, 1 \mathrm{H}), 3.08$ (s, 6H), 3.90 (q, $J=7.2$ $\mathrm{Hz}, 2 \mathrm{H}), 6.46 \sim 6.52(\mathrm{~m}, 2 \mathrm{H}), 6.89(\mathrm{~d}, J=7.6 \mathrm{~Hz}, 1 \mathrm{H})$, $6.97 \sim 7.02(\mathrm{~m}, 2 \mathrm{H}), 7.08(\mathrm{t}, J=7.6 \mathrm{~Hz}, 1 \mathrm{H}), 7.93 \sim 8.01$ $(\mathrm{m}, 2 \mathrm{H}) ;{ }^{13} \mathrm{C} \mathrm{NMR}\left(100 \mathrm{MHz}, \mathrm{CDCl}_{3}\right) \delta: 14.4,21.3,36.2$, 39.4, 58.5, 106.1, 125.4, 125.6, 127.6, 129.3, 137.1, 144.4, $146.5,155.0,180.5 ;{ }^{11} \mathrm{~B}$ NMR $\left(128.4 \mathrm{MHz}, \mathrm{CDCl}_{3}\right) \delta$ : -4.5 (br s, 1B); ESI-HRMS calcd for $\mathrm{C}_{19} \mathrm{H}_{27} \mathrm{BN}_{2} \mathrm{O}_{2} \mathrm{Na}$ $(\mathrm{M}+\mathrm{Na})^{+}$349.2063, found 349.2055.

(4-Dimethylaminopyridine)(1-ethoxy-3-(3-hydroxyphenyl)-1-oxopropan-2-yl)dihydroborate (3fa): The yield is $51 \%$ (50.4 mg as colorless liquid) after column chromatography. ${ }^{1} \mathrm{H}$ NMR $\left(400 \mathrm{MHz}, \mathrm{CDCl}_{3}\right) \delta: 1.05(\mathrm{t}, J=7.2$ $\mathrm{Hz}, 3 \mathrm{H}), 2.25 \sim 2.31(\mathrm{~m}, 1 \mathrm{H}), 2.61(\mathrm{dd}, J=3.2,14.4 \mathrm{~Hz}$, $1 \mathrm{H}), 2.84$ (dd, $J=10.8,14.0 \mathrm{~Hz}, 1 \mathrm{H}), 3.04$ (s, 6H), 3.91 (q, $J=7.2 \mathrm{~Hz}, 2 \mathrm{H}), 5.30$ (br s, $1 \mathrm{H}), 6.45$ (d, $J=7.2 \mathrm{~Hz}, 2 \mathrm{H})$, $6.51 \sim 6.80(\mathrm{~m}, 3 \mathrm{H}), 7.04(\mathrm{t}, J=8.0 \mathrm{~Hz}, 1 \mathrm{H}), 7.95(\mathrm{~d}, J=$ $7.2 \mathrm{~Hz}, 2 \mathrm{H}) ;{ }^{13} \mathrm{C}$ NMR $\left(100 \mathrm{MHz}, \mathrm{CDCl}_{3}\right) \delta: 14.4,34.0$, 39.5, 58.6, 106.1, 126.2, 126.4, 129.0, 130.6, 133.9, 141.7, 146.7, 155.1, 180.3; ${ }^{11} \mathrm{~B}$ NMR (128.4 MHz, $\left.\mathrm{CDCl}_{3}\right) \delta$ : -6.5 (br s, 1B); ESI-HRMS calcd for $\mathrm{C}_{18} \mathrm{H}_{25} \mathrm{BN}_{2} \mathrm{O}_{3} \mathrm{Na}$ $(\mathrm{M}+\mathrm{Na})^{+}$351.1856, found 351.1866.

(4-Dimethylaminopyridine)(1-ethoxy-3-([1,1'-biphenyl]4-yl)-1-oxopropan-2-yl)dihydroborate (3ga): The yield is $69 \%$ (80.0 $\mathrm{mg}$ as a white solid) after column chromatography. m.p. $131 \sim 133{ }^{\circ} \mathrm{C} ;{ }^{1} \mathrm{H}$ NMR $\left(400 \mathrm{MHz}, \mathrm{CDCl}_{3}\right) \delta$ : $1.06(\mathrm{t}, J=7.2 \mathrm{~Hz}, 3 \mathrm{H}), 2.27 \sim 2.34(\mathrm{~m}, 1 \mathrm{H}), 2.70(\mathrm{dd}, J=$ 3.6, $14.4 \mathrm{~Hz}, 1 \mathrm{H}), 2.97$ (dd, $J=10.4,14.4 \mathrm{~Hz}, 1 \mathrm{H}), 3.11$ (s, $6 \mathrm{H}), 3.92$ (q, $J=7.2 \mathrm{~Hz}, 2 \mathrm{H}), 6.51(\mathrm{~d}, J=7.2 \mathrm{~Hz}, 2 \mathrm{H})$, $7.26 \sim 7.32(\mathrm{~m}, 2 \mathrm{H}), 7.36 \sim 7.48(\mathrm{~m}, 5 \mathrm{H}), 7.56(\mathrm{~d}, J=7.2$ $\mathrm{Hz}, 2 \mathrm{H}), 8.01(\mathrm{~d}, J=7.2 \mathrm{~Hz}, 2 \mathrm{H}) ;{ }^{13} \mathrm{C} \mathrm{NMR}(100 \mathrm{MHz}$, $\left.\mathrm{CDCl}_{3}\right) \delta: 14.4,36.0,39.4,58.6,106.1,126.5,126.6$, $126.8,128.5,128.9,137.7,141.3,143.8,146.5,155.0$, $180.5 ;{ }^{11} \mathrm{~B}$ NMR $\left(128.4 \mathrm{MHz}, \mathrm{CDCl}_{3}\right) \delta$ : -5.0 (br s, 1B); ESI-HRMS calcd for $\mathrm{C}_{24} \mathrm{H}_{29} \mathrm{BN}_{2} \mathrm{O}_{2} \mathrm{Na} \quad(\mathrm{M}+\mathrm{Na})$ 411.2220, found 411.2222.

(4-Dimethylaminopyridine)(1-ethoxy-3-(4-fluorophenyl)-1-oxopropan-2-yl)dihydroborate (3ha): The yield is $73 \%$ (97.5 $\mathrm{mg}$ as a white solid) after column chromatography. m.p. 91 $92{ }^{\circ} \mathrm{C} ;{ }^{1} \mathrm{H}$ NMR $\left(400 \mathrm{MHz}, \mathrm{CDCl}_{3}\right) \delta$ : $1.01(\mathrm{t}, J=7.2 \mathrm{~Hz}, 3 \mathrm{H}), 2.15 \sim 2.24(\mathrm{~m}, 1 \mathrm{H}), 2.60(\mathrm{dd}, J=$ 4.0, 14.4 Hz, 1H), 2.88 (dd, $J=10.8,14.4 \mathrm{~Hz}, 1 \mathrm{H}), 3.07$ (s, $6 \mathrm{H}), 3.87(\mathrm{q}, J=7.2 \mathrm{~Hz}, 2 \mathrm{H}), 6.46 \sim 6.51(\mathrm{~m}, 2 \mathrm{H}), 6.81 \sim$ $6.88(\mathrm{~m}, 2 \mathrm{H}), 7.10 \sim 7.15(\mathrm{~m}, 2 \mathrm{H}), 7.92 \sim 7.98(\mathrm{~m}, 2 \mathrm{H})$; ${ }^{13} \mathrm{C} \mathrm{NMR}\left(100 \mathrm{MHz}, \mathrm{CDCl}_{3}\right) \delta: 14.4,35.5,39.4,58.5$, 
106.1, 114.3 (d, $J=20.7 \mathrm{~Hz}), 129.7$ (d, $J=7.5 \mathrm{~Hz}), 140.1$ $(\mathrm{d}, J=3.1 \mathrm{~Hz}), 146.5,155.0,160.7$ (d, $J=240.0 \mathrm{~Hz})$, 180.3; ${ }^{11} \mathrm{~B}$ NMR $\left(128.4 \mathrm{MHz}, \mathrm{CDCl}_{3}\right) \delta$ : -5.5 (br s, 1B); ${ }^{19} \mathrm{~F}$ NMR $\left(376 \mathrm{MHz}, \mathrm{CDCl}_{3}\right) \delta$ : -119.3 (s, 1F); ESIHRMS calcd for $\mathrm{C}_{18} \mathrm{H}_{24} \mathrm{BFN}_{2} \mathrm{O}_{2} \mathrm{Na}(\mathrm{M}+\mathrm{Na})^{+} 353.1813$, found 353.1810 .

(3-(4-Cyanophenyl)-1-ethoxy-1-oxopropan-2-yl)(4-dime thylaminopyridine)dihydroborate (3ia): The yield is $43 \%$ (42.8 $\mathrm{mg}$ as a white solid) after column chromatography. m.p. 91 92 ${ }^{\circ} \mathrm{C} ;{ }^{1} \mathrm{H}$ NMR $\left(400 \mathrm{MHz}, \mathrm{CDCl}_{3}\right) \delta$ : 1.01 (t, $J=7.2 \mathrm{~Hz}, 3 \mathrm{H}), 2.18 \sim 2.25(\mathrm{~m}, 1 \mathrm{H}), 2.69(\mathrm{dd}, J=4.0$, $14.4 \mathrm{~Hz}, 1 \mathrm{H}), 2.96(\mathrm{dd}, J=10.8,14.4 \mathrm{~Hz}, 1 \mathrm{H}), 3.11$ (s, $6 \mathrm{H}), 3.86(\mathrm{qd}, J=1.6,7.2 \mathrm{~Hz}, 2 \mathrm{H}), 6.48 \sim 6.54(\mathrm{~m}, 2 \mathrm{H})$, $7.28(\mathrm{~d}, J=8.4 \mathrm{~Hz}, 2 \mathrm{H}), 7.44 \sim 7.49(\mathrm{~m}, 2 \mathrm{H}), 7.91 \sim 7.98$ $(\mathrm{m}, 2 \mathrm{H}) ;{ }^{13} \mathrm{C}$ NMR $\left(100 \mathrm{MHz}, \mathrm{CDCl}_{3}\right) \delta: 14.4,36.7,39.5$, $58.7,106.2,108.6,119.5,129.3,131.7,146.6,150.5$, 155.1, 180.0; ${ }^{11} \mathrm{~B}$ NMR (128.4 MHz, $\mathrm{CDCl}_{3}$ ) $\delta: 5.1$ (br s, 1B); ESI-HRMS calcd for $\mathrm{C}_{19} \mathrm{H}_{24} \mathrm{BN}_{3} \mathrm{O}_{2} \mathrm{Na}(\mathrm{M}+\mathrm{Na})^{+}$ 360.1859 , found 360.1855 .

(3-(4-(Dimethylamino)phenyl)-1-ethoxy-1-oxopropan2-yl)(4-dimethylaminopyridine)dihydroborate (3ja): The yield is $49 \%$ (51.9 mg as colorless liquid) after column chromatography. ${ }^{1} \mathrm{H}$ NMR $\left(400 \mathrm{MHz}, \mathrm{CDCl}_{3}\right) \delta: 1.05$ (t, $J=7.2 \mathrm{~Hz}, 3 \mathrm{H}), 2.19 \sim 2.27(\mathrm{~m}, 1 \mathrm{H}), 2.56(\mathrm{dd}, J=4.0$, $14.4 \mathrm{~Hz}, 1 \mathrm{H}), 2.82 \sim 2.87(\mathrm{~m}, 1 \mathrm{H}), 2.86(\mathrm{~s}, 6 \mathrm{H}), 3.08(\mathrm{~s}$, $6 \mathrm{H}), 3.90$ (q, $J=7.2 \mathrm{~Hz}, 2 \mathrm{H}), 6.49$ (d, $J=7.6 \mathrm{~Hz}, 2 \mathrm{H})$, $6.61 \sim 6.65(\mathrm{~m}, 2 \mathrm{H}), 7.04 \sim 7.09(\mathrm{~m}, 2 \mathrm{H}), 7.94 \sim 8.01(\mathrm{~m}$, $2 \mathrm{H}) ;{ }^{13} \mathrm{C} \mathrm{NMR}\left(100 \mathrm{MHz}, \mathrm{CDCl}_{3}\right) \delta: 14.5,35.3,39.5,41.1$, $58.5,106.1,113.1,129.0,133.3,146.7,148.6,155.0$, $180.8 ;{ }^{11} \mathrm{~B}$ NMR $\left(128.4 \mathrm{MHz}, \mathrm{CDCl}_{3}\right) \delta$ : -4.5 (br s, 1B); ESI-HRMS calcd for $\mathrm{C}_{20} \mathrm{H}_{31} \mathrm{BN}_{3} \mathrm{O}_{2}(\mathrm{M}+\mathrm{H})^{+}$356.2509, found 356.2505 .

(4-Dimethylaminopyridine)(1-ethoxy-3-(naphthalen-2yl)-1-oxopropan-2-yl)dihydroborate (3ka): The yield is $64 \%$ (1.06 $\mathrm{g}$ as a white solid) after column chromatography. m.p. 89 92 ${ }^{\circ} \mathrm{C}$; ${ }^{1} \mathrm{H}$ NMR $\left(500 \mathrm{MHz}, \mathrm{CDCl}_{3}\right) \delta$ : $1.04(\mathrm{t}, J=6.8 \mathrm{~Hz}, 3 \mathrm{H}), 2.37 \sim 2.39(\mathrm{~m}, 1 \mathrm{H}), 2.82(\mathrm{dd}, J=$ $3.6,14.4 \mathrm{~Hz}, 1 \mathrm{H}), 3.00 \sim 3.13(\mathrm{~m}, 7 \mathrm{H}), 3.90(\mathrm{q}, J=7.2 \mathrm{~Hz}$, $2 \mathrm{H}), 6.45(\mathrm{~d}, J=6.4 \mathrm{~Hz}, 2 \mathrm{H}), 7.35 \sim 7.40(\mathrm{~m}, 3 \mathrm{H}), 7.61(\mathrm{~s}$, $1 \mathrm{H}), 7.66 \sim 7.75(\mathrm{~m}, 3 \mathrm{H}), 7.98(\mathrm{~d}, J=6.8 \mathrm{~Hz}, 2 \mathrm{H}) ;{ }^{13} \mathrm{C}$ NMR $\left(100 \mathrm{MHz}, \mathrm{CDCl}_{3}\right) \delta: 14.4,36.6,39.4,58.6,106.1$, $124.5,125.3,126.2,127.1,127.3,127.4,128.0,131.7$, 133.6, 142.1, 146.6, 155.0, 180.5; ${ }^{11} \mathrm{~B}$ NMR $(128.4 \mathrm{MHz}$, $\mathrm{CDCl}_{3}$ ) $\delta$ : -6.1 (br s, 1B); ESI-HRMS calcd for $\mathrm{C}_{22} \mathrm{H}_{27} \mathrm{~B}-$ $\mathrm{N}_{2} \mathrm{O}_{2} \mathrm{Na}(\mathrm{M}+\mathrm{Na})^{+}$385.2063, found 385.2063.

(4-Dimethylaminopyridine)(1-ethoxy-3-(1H-indol-6-yl)1-oxopropan-2-yl)dihydroborate (3la): The yield is $73 \%$ (76.4 mg as a white solid) after column chromatography. m.p. $146 \sim 148{ }^{\circ} \mathrm{C} ;{ }^{1} \mathrm{H}$ NMR (400 MHz, $\left.\mathrm{CDCl}_{3}\right) \delta: 1.06(\mathrm{t}$, $J=7.2 \mathrm{~Hz}, 3 \mathrm{H}), 2.31 \sim 2.38(\mathrm{~m}, 1 \mathrm{H}), 2.76(\mathrm{dd}, J=3.6$, $14.4 \mathrm{~Hz}, 1 \mathrm{H}), 3.00 \sim 3.07(\mathrm{~m}, 1 \mathrm{H}), 3.02(\mathrm{~s}, 6 \mathrm{H}), 3.87 \sim$ $3.95(\mathrm{~m}, 2 \mathrm{H}), 6.39 \sim 6.41(\mathrm{~m}, 1 \mathrm{H}), 6.41 \sim 6.45(\mathrm{~m}, 2 \mathrm{H})$, 7.05 (dd, $J=1.6,8.4 \mathrm{~Hz}, 1 \mathrm{H}), 7.08$ (t, $J=2.8 \mathrm{~Hz}, 1 \mathrm{H}), 7.19$ $(\mathrm{d}, J=8.0 \mathrm{~Hz}, 1 \mathrm{H}), 7.41 \sim 7.45(\mathrm{~m}, 1 \mathrm{H}), 7.95 \sim 8.00(\mathrm{~m}$, 2H), 8.19 (br s, $1 \mathrm{H}) ;{ }^{13} \mathrm{C}$ NMR $\left(100 \mathrm{MHz}, \mathrm{CDCl}_{3}\right) \delta: 14.5$, $36.5,39.3,58.5,102.0,106.0,110.3,119.7,123.3,123.7$, 127.8, 134.1, 135.7, 146.6, 155.0, 181.0; ${ }^{11} \mathrm{~B}$ NMR (128.4
$\left.\mathrm{MHz}, \mathrm{CDCl}_{3}\right) \delta$ : -5.9 (br s, 1B); ESI-HRMS calcd for $\mathrm{C}_{20} \mathrm{H}_{26} \mathrm{BN}_{3} \mathrm{O}_{2} \mathrm{Na}(\mathrm{M}+\mathrm{Na})^{+}$374.2016, found 374.2010.

(4-Dimethylaminopyridine)(1-ethoxy-3-(furan-2-yl)-1oxopropan-2-yl)dihydroborate (3ma): The yield is $57 \%$ (69.8 $\mathrm{mg}$ as a white solid) after column chromatography. m.p. $86 \sim 87{ }^{\circ} \mathrm{C} ;{ }^{1} \mathrm{H}$ NMR $\left(400 \mathrm{MHz}, \mathrm{CDCl}_{3}\right) \delta$ : $1.08(\mathrm{t}$, $J=7.2 \mathrm{~Hz}, 3 \mathrm{H}), 2.22 \sim 2.30(\mathrm{~m}, 1 \mathrm{H}), 2.59(\mathrm{dd}, J=4.0$, $16.0 \mathrm{~Hz}, 1 \mathrm{H}), 2.93(\mathrm{dd}, J=10.8,16.0 \mathrm{~Hz}, 1 \mathrm{H}), 3.09$ (s, $6 \mathrm{H}), 3.90 \sim 4.00(\mathrm{~m}, 2 \mathrm{H}), 5.89 \sim 5.93(\mathrm{~m}, 1 \mathrm{H}), 6.19(\mathrm{dd}$, $J=1.6,3.2 \mathrm{~Hz}, 1 \mathrm{H}), 6.47 \sim 6.52(\mathrm{~m}, 2 \mathrm{H}), 7.21(\mathrm{dd}, J=0.8$, $2.0 \mathrm{~Hz}, 1 \mathrm{H}), 7.93 \sim 7.99(\mathrm{~m}, 2 \mathrm{H}) ;{ }^{13} \mathrm{C} \mathrm{NMR}(100 \mathrm{MHz}$, $\left.\mathrm{CDCl}_{3}\right) \delta: 14.4,28.7,39.4,58.6,103.9,106.1,109.9$, $140.0,146.6,155.1,158.3,180.4 ;{ }^{11} \mathrm{~B}$ NMR (128.4 MHz, $\mathrm{CDCl}_{3}$ ) $\delta$ : -4.9 (br s, 1B); ESI-HRMS calcd for $\mathrm{C}_{16} \mathrm{H}_{23} \mathrm{~B}-$ $\mathrm{N}_{2} \mathrm{O}_{3} \mathrm{Na}(\mathrm{M}+\mathrm{Na})^{+}$325.1699, found 325.1702.

(4-Dimethylaminopyridine)(1-(tert-butoxy)-1-oxo-3-phe nylpropan-2-yl)dihydroborate (3na): The yield is $82 \%$ (83.6 $\mathrm{mg}$ as a white solid) after column chromatography. m.p. $79 \sim 80{ }^{\circ} \mathrm{C} ;{ }^{1} \mathrm{H}$ NMR $\left(400 \mathrm{MHz}, \mathrm{CDCl}_{3}\right) \delta$ : $1.25(\mathrm{~s}$, 9H), $2.11 \sim 2.18(\mathrm{~m}, 1 \mathrm{H}), 2.62(\mathrm{dd}, J=4.0,14.4 \mathrm{~Hz}, 1 \mathrm{H})$, $2.86(\mathrm{dd}, J=10.8,14.4 \mathrm{~Hz}, 1 \mathrm{H}), 3.09(\mathrm{~s}, 6 \mathrm{H}), 6.47 \sim 6.52$ $(\mathrm{m}, 2 \mathrm{H}), 7.04 \sim 7.10(\mathrm{~m}, 1 \mathrm{H}), 7.15 \sim 7.23(\mathrm{~m}, 4 \mathrm{H}), 7.97 \sim$ $8.04(\mathrm{~m}, 2 \mathrm{H}) ;{ }^{13} \mathrm{C}$ NMR $\left(100 \mathrm{MHz}, \mathrm{CDCl}_{3}\right) \delta: 28.2,36.6$, $39.4,106.0,124.8,127.6,128.5,144.8,146.7,155.0$, 180.2; ${ }^{11} \mathrm{~B}$ NMR (128.4 MHz, $\left.\mathrm{CDCl}_{3}\right) \delta$ : -4.8 (br s, 1B); ESI-HRMS calcd for $\mathrm{C}_{20} \mathrm{H}_{29} \mathrm{BN}_{2} \mathrm{O}_{2} \mathrm{Na}(\mathrm{M}+\mathrm{Na})^{+}$ 363.2220, found 363.2207.

(4-Dimethylaminopyridine)(1-(naphthalen-2-yloxy)-1oxo-3-phenylpropan-2-yl)dihydroborate (3oa): The yield is $74 \%$ (94.0 $\mathrm{mg}$ as a white solid) after column chromatography. m.p. 88 90 ${ }^{\circ} \mathrm{C}$; ${ }^{1} \mathrm{H}$ NMR $\left(400 \mathrm{MHz}, \mathrm{CDCl}_{3}\right) \delta$ : $2.51 \sim 2.61(\mathrm{~m}, 1 \mathrm{H}), 2.81(\mathrm{~d}, J=4.0,14.4 \mathrm{~Hz}, 1 \mathrm{H}), 3.00 \sim$ $3.08(\mathrm{~m}, 1 \mathrm{H}), 3.02(\mathrm{~s}, 6 \mathrm{H}), 6.44 \sim 6.51(\mathrm{~m}, 2 \mathrm{H}), 6.98(\mathrm{dd}$, $J=2.4,8.8 \mathrm{~Hz}, 1 \mathrm{H}), 7.15(\mathrm{tt}, J=1.6,7.2 \mathrm{~Hz}, 1 \mathrm{H}), 7.24 \sim$ $7.28(\mathrm{~m}, 3 \mathrm{H}), 7.29 \sim 7.33(\mathrm{~m}, 2 \mathrm{H}), 7.35 \sim 7.43(\mathrm{~m}, 2 \mathrm{H})$, $7.66 \sim 7.72(\mathrm{~m}, 2 \mathrm{H}), 7.74 \sim 7.77(\mathrm{~m}, 1 \mathrm{H}), 8.02 \sim 8.10(\mathrm{~m}$, $2 \mathrm{H}) ;{ }^{13} \mathrm{C} \mathrm{NMR}\left(100 \mathrm{MHz}, \mathrm{CDCl}_{3}\right) \delta: 36.5,39.4,106.3$, $118.4,122.1,125.0,125.2,126.0,127.4,127.6,128.0$, $128.6,128.7,131.0,133.8,144.2,146.6,149.4,155.1$, 179.1; ${ }^{11} \mathrm{~B}$ NMR $\left(128.4 \mathrm{MHz}, \mathrm{CDCl}_{3}\right) \delta$ : -4.5 (br s, 1B); ESI-HRMS calcd for $\mathrm{C}_{26} \mathrm{H}_{27} \mathrm{BN}_{2} \mathrm{O}_{2} \mathrm{Na} \quad(\mathrm{M}+\mathrm{Na}){ }^{+}$ 433.2063, found 433.2061.

(4-Dimethylaminopyridine)(2-oxochroman-3-yl)dihydroborate (3pa): The yield is $65 \%(73.9 \mathrm{mg}$ as a white solid) after column chromatography. m.p. $111 \sim 113{ }^{\circ} \mathrm{C} ;{ }^{1} \mathrm{H}$ NMR $\left(400 \mathrm{MHz}, \mathrm{CDCl}_{3}\right) \delta: 2.32 \sim 2.38(\mathrm{~m}, 1 \mathrm{H}), 2.69(\mathrm{~d}$, $J=15.6 \mathrm{~Hz}, 1 \mathrm{H}), 3.01(\mathrm{~s}, 6 \mathrm{H}), 3.17(\mathrm{dd}, J=6.4,15.6 \mathrm{~Hz}$, $1 \mathrm{H}), 6.39(\mathrm{~d}, J=7.6 \mathrm{~Hz}, 2 \mathrm{H}), 6.81 \sim 6.86(\mathrm{~m}, 1 \mathrm{H}), 6.94$ $(\mathrm{td}, J=1.2,7.4 \mathrm{~Hz}, 1 \mathrm{H}), 7.05 \sim 7.10(\mathrm{~m}, 2 \mathrm{H}), 7.81(\mathrm{~d}, J=$ $7.2 \mathrm{~Hz}, 2 \mathrm{H}) ;{ }^{13} \mathrm{C}$ NMR $\left(100 \mathrm{MHz}, \mathrm{CDCl}_{3}\right) \delta: 28.4,39.2$, $106.2,115.4,123.0,123.8,126.5,128.0,146.0,152.1$, 154.9, 176.8; ${ }^{11} \mathrm{~B}$ NMR $\left(128.4 \mathrm{MHz}, \mathrm{CDCl}_{3}\right) \delta$ : $-6.5(\mathrm{br}$ s, 1B); ESI-HRMS calcd for $\mathrm{C}_{16} \mathrm{H}_{19} \mathrm{BN}_{2} \mathrm{O}_{2} \mathrm{Na}(\mathrm{M}+\mathrm{Na})^{+}$ 305.1437, found 305.1440.

(1-(Cinnamyloxy)-1-oxo-3-phenylpropan-2-yl)(4-dimethylaminopyridine)dihydroborate (3qa): The yield is $39 \%$ (45.2 $\mathrm{mg}$ as a white solid) after column chromatog- 
raphy. m.p. 95 97 ${ }^{\circ} \mathrm{C} ;{ }^{1} \mathrm{H}$ NMR $\left(400 \mathrm{MHz}, \mathrm{CDCl}_{3}\right) \delta$ : $2.32 \sim 2.38(\mathrm{~m}, 1 \mathrm{H}), 2.71(\mathrm{~d}, J=3.6,14.4 \mathrm{~Hz}, 1 \mathrm{H}), 2.93(\mathrm{~s}$, $6 \mathrm{H}), 2.95 \sim 3.13(\mathrm{~m}, 1 \mathrm{H}), 4.43(\mathrm{ddd}, J=1.2,6.0,13.6 \mathrm{~Hz}$, $1 \mathrm{H}), 4.56$ (ddd, $J=1.2,6.0,13.6 \mathrm{~Hz}, 1 \mathrm{H}), 6.08$ (dt, $J=6.0$, $16.0 \mathrm{~Hz}, 1 \mathrm{H}), 6.36 \sim 6.43(\mathrm{~m}, 3 \mathrm{H}), 7.06 \sim 7.12(\mathrm{~m}, 1 \mathrm{H})$, $7.16 \sim 7.25(\mathrm{~m}, 5 \mathrm{H}), 7.27 \sim 7.32(\mathrm{~m}, 4 \mathrm{H}), 7.96(\mathrm{~d}, J=7.2$ $\mathrm{Hz}, 2 \mathrm{H}) ;{ }^{13} \mathrm{C} \mathrm{NMR}\left(100 \mathrm{MHz}, \mathrm{CDCl}_{3}\right) \delta: 36.4,39.2,63.2$, $106.1,124.9,125.0,126.4,127.6,127.9,128.46,128.54$, $132.1,136.7,144.5,146.6,154.9,180.2 ;{ }^{11} \mathrm{~B}$ NMR $(128.4$ $\left.\mathrm{MHz}, \mathrm{CDCl}_{3}\right) \delta$ : -6.0 (br s, 1B); ESI-HRMS calcd for $\mathrm{C}_{25} \mathrm{H}_{29} \mathrm{BN}_{2} \mathrm{O}_{2} \mathrm{Na}(\mathrm{M}+\mathrm{Na})^{+}$423.2214, found 423.2213.

(4-Dimethylaminopyridine)(1-ethoxy-1-oxo-3,3-diphenylpropan-2-yl)dihydroborate (3ra): The yield is $51 \%$ (59.4 $\mathrm{mg}$ as a white solid) after column chromatography. m.p. $125 \sim 127{ }^{\circ} \mathrm{C} ;{ }^{1} \mathrm{H}$ NMR $\left(400 \mathrm{MHz}, \mathrm{CDCl}_{3}\right) \delta: 0.92(\mathrm{t}$, $J=6.4 \mathrm{~Hz}, 3 \mathrm{H}), 2.85 \sim 2.92(\mathrm{~m}, 1 \mathrm{H}), 3.06(\mathrm{~s}, 6 \mathrm{H}), 3.82(\mathrm{q}$, $J=6.4 \mathrm{~Hz}, 2 \mathrm{H}), 4.18$ (d, $J=9.6 \mathrm{~Hz}, 1 \mathrm{H}), 6.36$ (d, $J=5.2$ $\mathrm{Hz}, 2 \mathrm{H}), 7.03(\mathrm{dt}, J=6.0,12.4 \mathrm{~Hz}, 2 \mathrm{H}), 7.13 \sim 7.17(\mathrm{~m}$, $4 \mathrm{H}), 7.27(\mathrm{~d}, J=6.0 \mathrm{~Hz}, 2 \mathrm{H}), 7.33(\mathrm{~d}, J=6.4 \mathrm{~Hz}, 2 \mathrm{H}), 7.73$ $(\mathrm{d}, J=5.6 \mathrm{~Hz}, 2 \mathrm{H}) ;{ }^{13} \mathrm{C} \mathrm{NMR}\left(100 \mathrm{MHz}, \mathrm{CDCl}_{3}\right) \delta: 14.2$, $39.4,53.0,58.6,105.9,125.2,125.3,127.4,127.90$, $127.94,128.5,145.7,146.7,147.6,154,9,179.9 ;{ }^{11} \mathrm{~B}$ NMR $\left(128.4 \mathrm{MHz}, \mathrm{CDCl}_{3}\right) \delta$ : -6.9 (br s, 1B); ESI-HRMS calcd for $\mathrm{C}_{24} \mathrm{H}_{29} \mathrm{BN}_{2} \mathrm{O}_{2} \mathrm{Na}(\mathrm{M}+\mathrm{Na})^{+} 411.2220$, found 411.2219.

(4-Dimethylaminopyridine)(1-ethoxy-2-methyl-1-oxo-3phenylpropan-2-yl)dihydroborate (3sa): The yield is $64 \%$ (64.3 $\mathrm{mg}$ as a white solid) after column chromatography (eluent: petroleum ether). m.p. 62 $65{ }^{\circ} \mathrm{C} ;{ }^{1} \mathrm{H}$ NMR $(500$ $\left.\mathrm{MHz}, \mathrm{CDCl}_{3}\right) \delta: 0.92(\mathrm{~s}, 3 \mathrm{H}), 1.18(\mathrm{t}, J=5.6 \mathrm{~Hz}, 3 \mathrm{H}), 2.44$ $(\mathrm{d}, J=10.8 \mathrm{~Hz}, 1 \mathrm{H}), 3.10(\mathrm{~s}, 6 \mathrm{H}), 3.31(\mathrm{~d}, J=10.8 \mathrm{~Hz}$, $1 \mathrm{H}), 4.00(\mathrm{q}, J=5.6 \mathrm{~Hz}, 2 \mathrm{H}), 6.50(\mathrm{~d}, J=5.6 \mathrm{~Hz}, 2 \mathrm{H})$, $7.07 \sim 7.11(\mathrm{~m}, 1 \mathrm{H}), 7.14 \sim 7.19(\mathrm{~m}, 4 \mathrm{H}), 7.94(\mathrm{~d}, J=5.6$ $\mathrm{Hz}, 2 \mathrm{H}) ;{ }^{13} \mathrm{C}$ NMR $\left(100 \mathrm{MHz}, \mathrm{CDCl}_{3}\right) \delta: 14.5,20.0,39.4$, 43.1, 58.7, 105.8, 125.1, 127.5, 130.0, 142.1, 147.0, 155.1, 181.6; ${ }^{11} \mathrm{~B}$ NMR $\left(128.4 \mathrm{MHz}, \mathrm{CDCl}_{3}\right) \delta$ : -2.2 (br s, 1B); ESI-HRMS calcd for $\mathrm{C}_{19} \mathrm{H}_{27} \mathrm{BN}_{2} \mathrm{O}_{2} \mathrm{Na}(\mathrm{M}+\mathrm{Na})^{+}$ 349.2063, found 349.2060. Meanwhile, (4-dimethylaminopyridine)(3-ethoxy-2-methyl-3-oxo-1-phenylpropyl)dihydroborate (3sa') was obtained in 5\% yield $(5.02 \mathrm{mg}$ as colorless liquid) after column chromatography. ${ }^{1} \mathrm{H}$ NMR $\left(500 \mathrm{MHz}, \mathrm{CDCl}_{3}\right) \delta: 0.86(\mathrm{t}, J=5.6 \mathrm{~Hz}, 3 \mathrm{H}), 1.38(\mathrm{~d}, J=$ $5.2 \mathrm{~Hz}, 3 \mathrm{H}), 2.08 \sim 2.12(\mathrm{~m}, 1 \mathrm{H}), 2.79 \sim 2.86(\mathrm{~m}, 1 \mathrm{H}), 3.02$ $(\mathrm{s}, 6 \mathrm{H}), 3.77(\mathrm{q}, J=5.6 \mathrm{~Hz}, 2 \mathrm{H}), 6.26(\mathrm{~d}, J=5.6 \mathrm{~Hz}, 2 \mathrm{H})$, $6.86 \sim 6.88(\mathrm{~m}, 3 \mathrm{H}), 6.97 \sim 7.00(\mathrm{~m}, 2 \mathrm{H}), 7.58(\mathrm{~d}, J=6.0$ $\mathrm{Hz}, 2 \mathrm{H}) ;{ }^{13} \mathrm{C} \mathrm{NMR}\left(100 \mathrm{MHz}, \mathrm{CDCl}_{3}\right) \delta: 13.9,17.8,29.7$, 39.4, 44.7, 59.1, 105.7, 123.0, 127.1, 128.1, 146.3, 150.0, 154.6, 178.8; ${ }^{11} \mathrm{~B}$ NMR (128.4 MHz, $\left.\mathrm{CDCl}_{3}\right) \delta$ : -2.2 (br s, 1B); ESI-HRMS calcd for $\mathrm{C}_{19} \mathrm{H}_{27} \mathrm{BN}_{2} \mathrm{O}_{2} \mathrm{Na}(\mathrm{M}+\mathrm{Na})^{+}$ 349.2063, found 349.2060.

(1-(Diallylamino)-1-oxo-3-phenylpropan-2-yl)(4-dimethylaminopyridine)dihydroborate (3ta): The yield is $66 \%$ (71.4 mg as a white solid) after column chromatography. m.p. $126 \sim 129{ }^{\circ} \mathrm{C} ;{ }^{1} \mathrm{H}$ NMR $\left(400 \mathrm{MHz}, \mathrm{CDCl}_{3}\right) \delta: 2.35 \sim$ $2.43(\mathrm{~m}, 1 \mathrm{H}), 2.54(\mathrm{dd}, J=3.6,13.2 \mathrm{~Hz}, 1 \mathrm{H}), 2.83(\mathrm{dd}, J=$ 10.4, $13.2 \mathrm{~Hz}, 1 \mathrm{H}), 3.09$ (s, 6H), 3.36 (dd, $J=6.0,15.2 \mathrm{~Hz}$, $1 \mathrm{H}), 3.56(\mathrm{dd}, J=5.2,17.6 \mathrm{~Hz}, 1 \mathrm{H}), 4.20 \sim 4.30(\mathrm{~m}, 1 \mathrm{H})$, $4.30 \sim 4.40(\mathrm{~m}, 1 \mathrm{H}), 4.69(\mathrm{dd}, J=1.6,17.2 \mathrm{~Hz}, 1 \mathrm{H}), 4.85$ $(\mathrm{dd}, J=1.2,10.4 \mathrm{~Hz}, 1 \mathrm{H}), 5.02 \sim 5.12(\mathrm{~m}, 2 \mathrm{H}), 5.48 \sim 5.61$ $(\mathrm{m}, 1 \mathrm{H}), 5.67 \sim 5.79(\mathrm{~m}, 1 \mathrm{H}), 6.46 \sim 6.53(\mathrm{~m}, 2 \mathrm{H}), 7.00 \sim$ $7.09(\mathrm{~m}, 1 \mathrm{H}), 7.11 \sim 7.18(\mathrm{~m}, 4 \mathrm{H}), 7.92 \sim 8.01(\mathrm{~m}, 2 \mathrm{H})$; ${ }^{13} \mathrm{C}$ NMR $\left(100 \mathrm{MHz}, \mathrm{CDCl}_{3}\right) \delta: 37.1,39.3,47.0,48.8$, $105.8,115.2,115.4,124.7,127.6,128.8,134.1,135.0$, 145.0, 146.9, 155.1, 180.0; ${ }^{11} \mathrm{~B}$ NMR (128.4 $\left.\mathrm{MHz} \mathrm{CDCl}_{3}\right)$ $\delta$ : -5.4 (br s, 1B); ESI-HRMS calcd for $\mathrm{C}_{22} \mathrm{H}_{30} \mathrm{BN}_{3} \mathrm{ONa}$ $(\mathrm{M}+\mathrm{Na})^{+}$386.2374, found 386.2371.

(4-Dimethylaminopyridine)(1-((4-methyl- $N$-(prop-2-yn1-yl)phenyl)sulfonamido)-1-oxo-3-phenylpropan-2-yl)dihydroborate (3ua): The yield is $49 \%$ ( $70.5 \mathrm{mg}$ as a white solid) after column chromatography. m.p. $120 \sim 122{ }^{\circ} \mathrm{C}$; ${ }^{1} \mathrm{H}$ NMR (400 MHz, $\left.\mathrm{CDCl}_{3}\right) \delta: 2.09(\mathrm{t}, J=2.4 \mathrm{~Hz}, 1 \mathrm{H})$, 2.38 (s, 3H), 2.54 (dd, $J=3.6,13.6 \mathrm{~Hz}, 1 \mathrm{H}), 2.65$ (dd, $J=$ 10.0, 13.6 Hz, 1H), 2.75 2.77 (m, 1H), $3.09(\mathrm{~s}, 6 \mathrm{H}), 4.70$ (dd, $J=2.4,18.8 \mathrm{~Hz}, 1 \mathrm{H}), 4.88(\mathrm{dd}, J=2.4,18.8 \mathrm{~Hz}$, $1 \mathrm{H}), 6.47(\mathrm{~d}, J=7.6 \mathrm{~Hz}, 2 \mathrm{H}), 6.88 \sim 6.90(\mathrm{~m}, 2 \mathrm{H}), 6.96 \sim$ $6.99(\mathrm{~m}, 3 \mathrm{H}), 7.14$ (d, $J=8.4 \mathrm{~Hz}, 2 \mathrm{H}), 7.75$ (d, $J=8.8 \mathrm{~Hz}$, 2H), $7.84(\mathrm{~d}, J=8.0 \mathrm{~Hz}, 2 \mathrm{H}) ;{ }^{13} \mathrm{C} \mathrm{NMR}\left(100 \mathrm{MHz}, \mathrm{CDCl}_{3}\right)$ $\delta: 21.5,35.2,35.9,39.4,72.2,79.4,106.1,124.7,127.5$, $128.0,128.6,128.8,137.4,143.1,143.6,146.9,155.2$, 179.1; ${ }^{11} \mathrm{~B}$ NMR $\left(128.4 \mathrm{MHz}, \mathrm{CDCl}_{3}\right) \delta$ : -7.3 (br s, 1B); ESI- HRMS calcd for $\mathrm{C}_{26} \mathrm{H}_{30} \mathrm{BN}_{3} \mathrm{O}_{3} \mathrm{SNa}(\mathrm{M}+\mathrm{Na})$ 498.1993, found $m / z$ 498.1985.

(3-(3-(Allyloxy)phenyl)-1-morpholino-1-oxopropan-2yl)(4-dimethylaminopyridine)dihydroborate (3va): The yield is $62 \%(63.9 \mathrm{mg}$ as a white solid) after column chromatography. m.p. $132 \sim 133{ }^{\circ} \mathrm{C}$; ${ }^{1} \mathrm{H}$ NMR (400 MHz, $\left.\mathrm{CDCl}_{3}\right) \delta: 2.40 \sim 2.46(\mathrm{~m}, 1 \mathrm{H}), 2.51(\mathrm{dd}, J=3.6,14.0 \mathrm{~Hz}$, $1 \mathrm{H}), 2.85(\mathrm{dd}, J=10.0,14.0 \mathrm{~Hz}, 1 \mathrm{H}), 3.10(\mathrm{~s}, 6 \mathrm{H}), 3.30 \sim$ $3.70(\mathrm{~m}, 8 \mathrm{H}), 4.47(\mathrm{dt}, J=1.6,5.2 \mathrm{~Hz}, 2 \mathrm{H}), 5.24(\mathrm{dq}, J=$ 1.6, $10.4 \mathrm{~Hz}, 1 \mathrm{H}), 5.37$ (dq, $J=1.6,17.2 \mathrm{~Hz}, 1 \mathrm{H}), 5.98 \sim$ $6.09(\mathrm{~m}, 1 \mathrm{H}), 6.46 \sim 6.53(\mathrm{~m}, 2 \mathrm{H}), 6.70 \sim 6.77(\mathrm{~m}, 2 \mathrm{H})$, $7.01 \sim 7.07(\mathrm{~m}, 2 \mathrm{H}), 7.91 \sim 7.98(\mathrm{~m}, 2 \mathrm{H}) ;{ }^{13} \mathrm{C}$ NMR $(100$ $\left.\mathrm{MHz}, \mathrm{CDCl}_{3}\right) \delta: 35.8,39.4,67.1,67.2,67.3,67.4,68.8$, $106.0,114.2,117.3,129.3,133.7,137.4,146.8,155.1$, $156.2,179.1 ;{ }^{11} \mathrm{~B}$ NMR $\left(128.4 \mathrm{MHz}, \mathrm{CDCl}_{3}\right) \delta$ : $-7.2(\mathrm{br}$ s, 1B); ESI-HRMS calcd for $\mathrm{C}_{23} \mathrm{H}_{32} \mathrm{BN}_{3} \mathrm{O}_{3} \mathrm{Na}(\mathrm{M}+\mathrm{Na})^{+}$ 432.2434, found 432.2434 .

(1-(Dibenzylamino)-1-oxo-3-phenylpropan-2-yl)(4-dimethylaminopyridine)dihydroborate (3wa): The yield is $65 \%$ (88.8 $\mathrm{mg}$ as a white solid) after column chromatography. m.p. $112 \sim 115{ }^{\circ} \mathrm{C}$; ${ }^{1} \mathrm{H}$ NMR $\left(400 \mathrm{MHz}, \mathrm{CDCl}_{3}\right) \delta$ : $2.49 \sim 2.60(\mathrm{~m}, 2 \mathrm{H}), 2.94(\mathrm{dd}, J=10.4,12.4 \mathrm{~Hz}, 1 \mathrm{H}), 3.06$ (s, 6H), 3.67 (d, $J=14.8 \mathrm{~Hz}, 1 \mathrm{H}), 4.12(\mathrm{~d}, J=17.2 \mathrm{~Hz}$, $1 \mathrm{H}), 4.93$ (d, $J=16.8 \mathrm{~Hz}, 1 \mathrm{H}), 5.14$ (d, $J=14.8 \mathrm{~Hz}, 1 \mathrm{H})$, $6.35 \sim 6.41(\mathrm{~m}, 2 \mathrm{H}), 6.75(\mathrm{~d}, J=6.8 \mathrm{~Hz}, 2 \mathrm{H}), 7.08 \sim 7.19$ $(\mathrm{m}, 8 \mathrm{H}), 7.22 \sim 7.31(\mathrm{~m}, 5 \mathrm{H}), 7.85 \sim 7.90(\mathrm{~m}, 2 \mathrm{H}) ;{ }^{13} \mathrm{C}$ NMR (100 MHz, $\left.\mathrm{CDCl}_{3}\right) \delta: 37.3,39.3,47.6,49.9,105.9$, $124.8,126.48,126.53,126.6,127.8,128.1,128.27,128.34$, $129.0,137.8,139.3,145.1,146.9,155.0,180.0 ;{ }^{11} \mathrm{~B}$ NMR $\left(128.4 \mathrm{MHz}, \mathrm{CDCl}_{3}\right) \delta$ : -5.1 (br s, 1B); ESI-HRMS calcd for $\mathrm{C}_{30} \mathrm{H}_{34} \mathrm{BN}_{3} \mathrm{ONa}(\mathrm{M}+\mathrm{Na})^{+} 486.2693$, found 486.2688 .

(4-Dimethylaminopyridine)(1-oxo-1-(2-oxopyrrolidin-1yl)-3-phenylpropan-2-yl)dihydroborate (3xa): The yield is $22 \%$ (24.3 $\mathrm{mg}$ as a white solid) after column chromatography. m.p. $145 \sim 146{ }^{\circ} \mathrm{C} ;{ }^{1} \mathrm{H}$ NMR $\left(400 \mathrm{MHz}, \mathrm{CDCl}_{3}\right) \delta$ : 
$1.81 \sim 1.91(\mathrm{~m}, 2 \mathrm{H}), 2.46 \sim 2.55(\mathrm{~m}, 3 \mathrm{H}), 3.10(\mathrm{~s}, 6 \mathrm{H})$, $3.15(\mathrm{dd}, J=10.0,14.4 \mathrm{~Hz}, 1 \mathrm{H}), 3.70 \sim 3.81(\mathrm{~m}, 2 \mathrm{H})$, $3.85 \sim 3.94(\mathrm{~m}, 1 \mathrm{H}), 6.45 \sim 6.52(\mathrm{~m}, 2 \mathrm{H}), 6.98 \sim 7.06(\mathrm{~m}$, $1 \mathrm{H}), 7.09 \sim 7.16(\mathrm{~m}, 4 \mathrm{H}), 8.07 \sim 8.17(\mathrm{~m}, 2 \mathrm{H}) ;{ }^{13} \mathrm{C} \mathrm{NMR}$ $\left(100 \mathrm{MHz}, \mathrm{CDCl}_{3}\right) \delta: 17.0,34.6,36.0,39.4,46.0,106.1$, $124.7,127.7,128.6,144.5,146.8,155.0,174.8,180.9 ;{ }^{11} \mathrm{~B}$ NMR (128.4 MHz, $\left.\mathrm{CDCl}_{3}\right) \delta$ : -4.9 (br s, 1B); ESIHRMS calcd for $\mathrm{C}_{20} \mathrm{H}_{26} \mathrm{BN}_{3} \mathrm{O}_{2} \mathrm{Na}(\mathrm{M}+\mathrm{Na})^{+} 374.2016$, found 374.2016 .

(1-Carboxy-2-phenylethyl)(4-dimethylaminopyridine)dihydroborate (3ya): The yield is $76 \%(102.8 \mathrm{mg}$ as a white solid) after column chromatography. m.p. 138 $139{ }^{\circ} \mathrm{C} ;{ }^{1} \mathrm{H}$ NMR $\left(400 \mathrm{MHz}, \mathrm{CDCl}_{3}\right) \delta: 2.23 \sim 2.31(\mathrm{~m}$, $1 \mathrm{H}), 2.68(\mathrm{dd}, J=3.6,14.4 \mathrm{~Hz}, 1 \mathrm{H}), 2.91(\mathrm{dd}, J=10.4$, $14.4 \mathrm{~Hz}, 1 \mathrm{H}), 3.06(\mathrm{~s}, 6 \mathrm{H}), 6.41(\mathrm{~d}, J=7.2 \mathrm{~Hz}, 2 \mathrm{H}), 7.07 \sim$ $7.14(\mathrm{~m}, 1 \mathrm{H}), 7.17 \sim 7.25(\mathrm{~m}, 4 \mathrm{H}), 7.95(\mathrm{~d}, J=7.6 \mathrm{~Hz}$, $2 \mathrm{H}) ;{ }^{13} \mathrm{C}$ NMR $\left(100 \mathrm{MHz}, \mathrm{CDCl}_{3}\right) \delta: 36.1,39.5,106.3$, $125.1,127.9,128.5,144.4,146.5,155.1,185.2 ;{ }^{11} \mathrm{~B}$ NMR $\left(128.4 \mathrm{MHz} \mathrm{CDCl}_{3}\right) \delta$ : -24.4 (br s, 1B); ESI-HRMS calcd for $\mathrm{C}_{16} \mathrm{H}_{21} \mathrm{BN}_{2} \mathrm{O}_{2} \mathrm{Na}(\mathrm{M}+\mathrm{Na})^{+}$307.1594, found 307.1590 .

(4-Dimethylaminopyridine)(1-ethoxy-3-methyl-1-oxobutan-2-yl)dihydroborate (3za): The yield is $17 \%(21.4 \mathrm{mg}$ as a white solid) after column chromatography. m.p. 65 $68{ }^{\circ} \mathrm{C} ;{ }^{1} \mathrm{H}$ NMR $\left(400 \mathrm{MHz}, \mathrm{CDCl}_{3}\right) \delta: 0.91(\mathrm{~d}, J=6.4 \mathrm{~Hz}$, $3 \mathrm{H}), 0.98(\mathrm{~d}, J=6.8 \mathrm{~Hz}, 3 \mathrm{H}), 1.08(\mathrm{t}, J=7.2 \mathrm{~Hz}, 3 \mathrm{H})$, $1.55 \sim 1.63(\mathrm{~m}, 1 \mathrm{H}), 1.76 \sim 1.85(\mathrm{~m}, 1 \mathrm{H}), 3.09(\mathrm{~s}, 6 \mathrm{H})$, $3.82 \sim 3.97(\mathrm{~m}, 2 \mathrm{H}), 6.45 \sim 6.51(\mathrm{~m}, 2 \mathrm{H}), 7.95 \sim 0.02(\mathrm{~m}$, $2 \mathrm{H}) ;{ }^{13} \mathrm{C} \mathrm{NMR}\left(100 \mathrm{MHz}, \mathrm{CDCl}_{3}\right) \delta: 14.5,22.0,23.7,29.0$, $39.4,58.3,106.0,146.7,155.0,181.3 ;{ }^{11} \mathrm{~B}$ NMR $(128.4$ $\mathrm{MHz}, \mathrm{CDCl}_{3}$ ) $\delta$ : -5.9 (br s, 1B); ESI-HRMS calcd for $\mathrm{C}_{14} \mathrm{H}_{25} \mathrm{BN}_{2} \mathrm{O}_{2} \mathrm{Na}(\mathrm{M}+\mathrm{Na})^{+}$287.1907, found 287.1909.

(1-Cyano-2-phenylethyl)(4-dimethylaminopyridine)dihydroborate (5a): The yield is $65 \%(71.9 \mathrm{mg}$ as a white solid) after column chromatography. m.p. 93 $\sim 95{ }^{\circ} \mathrm{C} ;{ }^{1} \mathrm{H}$ NMR $\left(400 \mathrm{MHz}, \mathrm{CDCl}_{3}\right) \delta: 1.93 \sim 2.02(\mathrm{~m}, 1 \mathrm{H}), 2.64(\mathrm{dd}$, $J=11.2,14.0 \mathrm{~Hz}, 1 \mathrm{H}), 2.80(\mathrm{dd}, J=4.8,14.0 \mathrm{~Hz}, 1 \mathrm{H})$, $3.09(\mathrm{~s}, 6 \mathrm{H}), 6.54(\mathrm{~d}, J=7.6 \mathrm{~Hz}, 2 \mathrm{H}), 7.14 \sim 7.20(\mathrm{~m}, 1 \mathrm{H})$, $7.23 \sim 7.31(\mathrm{~m}, 4 \mathrm{H}), 8.09(\mathrm{~d}, J=7.2 \mathrm{~Hz}, 2 \mathrm{H}) ;{ }^{13} \mathrm{C} \mathrm{NMR}$ $\left(100 \mathrm{MHz}, \mathrm{CDCl}_{3}\right) \delta: 36.8,39.4,106.5,125.8,128.08$, 128.13, 128.4, 142.1, 146.4, 155.2; ${ }^{11} \mathrm{~B}$ NMR (128.4 MHz, $\mathrm{CDCl}_{3}$ ) $\delta$ : -6.3 (br s, 1B); ESI-HRMS calcd for $\mathrm{C}_{16} \mathrm{H}_{20} \mathrm{~B}-$ $\mathrm{N}_{3} \mathrm{Na}(\mathrm{M}+\mathrm{Na})^{+}$288.1648, found 288.1650.

(4-Dimethylaminopyridine)(1,1,1-trifluoro-3-(3,4,5trimethoxyphenyl)propan-2-yl)dihydroborate $\mathbf{( 5 b ) : ~ T h e ~}$ yield is $85 \%$ (141.2 $\mathrm{mg}$ as colorless liquid) after column chromatography. ${ }^{1} \mathrm{H}$ NMR $\left(400 \mathrm{MHz}, \mathrm{CDCl}_{3}\right) \delta: 1.63$ (br s, $1 \mathrm{H}), 2.53(\mathrm{dd}, J=8.8,13.6 \mathrm{~Hz}, 1 \mathrm{H}), 2.86(\mathrm{dd}, J=4.8,14.0$ $\mathrm{Hz}, 1 \mathrm{H}), 3.08(\mathrm{~s}, 6 \mathrm{H}), 3.81(\mathrm{~s}, 3 \mathrm{H}), 3.82(\mathrm{~s}, 6 \mathrm{H}), 6.38 \sim$ $6.46(\mathrm{~m}, 4 \mathrm{H}), 7.85(\mathrm{~d}, J=7.6 \mathrm{~Hz}, 2 \mathrm{H}) ;{ }^{13} \mathrm{C}$ NMR $(100$ $\left.\mathrm{MHz}, \mathrm{CDCl}_{3}\right) \delta: 34.7(\mathrm{q}, J=4.0 \mathrm{~Hz}), 39.4,55.9,60.8$, $105.9,106.1,132.4$ (q, $J=279.0 \mathrm{~Hz}), 135.5,139.0,146.6$, 152.6, 154.9; ${ }^{11} \mathrm{~B}$ NMR $\left(128.4 \mathrm{MHz}, \mathrm{CDCl}_{3}\right) \delta$ : $-7.7(\mathrm{br}$ $\mathrm{s}, 1 \mathrm{~B}) ;{ }^{19} \mathrm{~F}$ NMR $\left(376 \mathrm{MHz}, \mathrm{CDCl}_{3}\right) \delta:-63.6(\mathrm{~d}, J=12.4$ $\mathrm{Hz}, 3 \mathrm{~F})$; ESI-HRMS calcd for $\mathrm{C}_{19} \mathrm{H}_{26} \mathrm{BF}_{3} \mathrm{~N}_{2} \mathrm{O}_{3} \mathrm{Na}(\mathrm{M}+$ $\mathrm{Na})^{+}$421.1886, found 421.1897.

(4-Dimethylaminopyridine)(2-phenyl-1-(phenylsul- fonyl)ethyl)dihydroborate (5c): The yield is $25 \%$ (28.4 mg as a white solid) after column chromatography (eluent: petroleum ether). m.p. $128 \sim 130{ }^{\circ} \mathrm{C}$; ${ }^{1} \mathrm{H}$ NMR $(400 \mathrm{MHz}$, $\left.\mathrm{CDCl}_{3}\right) \delta: 2.65(\mathrm{dd}, J=8.0,10.4 \mathrm{~Hz}, 1 \mathrm{H}), 2.73 \sim 2.79(\mathrm{~m}$, $1 \mathrm{H}), 2.89$ (dd, $J=1.2,10.4 \mathrm{~Hz}, 1 \mathrm{H}), 3.08$ (s, 6H), 6.44 (d, $J=5.6 \mathrm{~Hz}, 2 \mathrm{H}), 6.93(\mathrm{~d}, J=5.6 \mathrm{~Hz}, 2 \mathrm{H}), 7.08(\mathrm{t}, J=5.6$ $\mathrm{Hz}, 1 \mathrm{H}), 7.13(\mathrm{t}, J=6.0 \mathrm{~Hz}, 2 \mathrm{H}), 7.45(\mathrm{t}, J=8.0 \mathrm{~Hz}, 2 \mathrm{H})$, $7.51(\mathrm{t}, J=5.6 \mathrm{~Hz}, 1 \mathrm{H}), 7.86(\mathrm{~d}, J=6.0 \mathrm{~Hz}, 2 \mathrm{H}), 7.96(\mathrm{~d}$, $J=5.6 \mathrm{~Hz}, 2 \mathrm{H}) ;{ }^{13} \mathrm{C}$ NMR $\left(100 \mathrm{MHz}, \mathrm{CDCl}_{3}\right) \delta: 34.8$, $39.4,106.1,125.5,127.9,128.2,128.4,128.9,131.9$, $140.9,141.5,147.3,155.1 ;{ }^{11} \mathrm{~B}$ NMR $\left(128.4 \mathrm{MHz}, \mathrm{CDCl}_{3}\right)$ $\delta$ : -10.1 (br s, 1B); ESI-HRMS calcd for $\mathrm{C}_{21} \mathrm{H}_{25} \mathrm{BN}_{2}$ $\mathrm{O}_{2} \mathrm{SNa}(\mathrm{M}+\mathrm{Na})^{+} 403.1627$, found 403.1626 .

(4-Dimethylaminopyridine)(1-(diethoxyphosphoryl)-2-p henylethyl)dihydroborate (5d): The yield is $15 \%(25.7 \mathrm{mg}$ as colorless liquid) after column chromatography. ${ }^{1} \mathrm{H}$ NMR $\left(400 \mathrm{MHz}, \mathrm{CDCl}_{3}\right) \delta: 1.00(\mathrm{t}, J=7.0 \mathrm{~Hz}, 3 \mathrm{H}), 1.14(\mathrm{t}, J=$ $7.0 \mathrm{~Hz}, 3 \mathrm{H}), 1.84 \sim 1.86(\mathrm{~m}, 1 \mathrm{H}), 2.08 \sim 2.22(\mathrm{~m}, 1 \mathrm{H})$, $2.32 \sim 2.37(\mathrm{~m}, 1 \mathrm{H}), 3.04(\mathrm{~s}, 6 \mathrm{H}), 3.53 \sim 3.61(\mathrm{~m}, 1 \mathrm{H})$, $3.73 \sim 3.77(\mathrm{~m}, 1 \mathrm{H}), 3.80 \sim 3.94(\mathrm{~m}, 2 \mathrm{H}), 6.30(\mathrm{~d}, J=6.8$ $\mathrm{Hz}, 2 \mathrm{H}), 6.90 \sim 6.94(\mathrm{~m}, 3 \mathrm{H}), 7.04 \sim 7.07(\mathrm{~m}, 2 \mathrm{H}), 7.63(\mathrm{~d}$, $J=6.8 \mathrm{~Hz}, 2 \mathrm{H}) ;{ }^{13} \mathrm{C} \mathrm{NMR}\left(100 \mathrm{MHz}, \mathrm{CDCl}_{3}\right) \delta: 16.4(\mathrm{~d}$, $J=5.1 \mathrm{~Hz}), 29.3,29.7,61.8(\mathrm{~d}, J=4.2 \mathrm{~Hz}), 113.2,114.7$, $127.7,128.8,130.2,134.8(\mathrm{~d}, J=18.4 \mathrm{~Hz}), 148.8(\mathrm{~d}, J=$ $5.2 \mathrm{~Hz}) ;{ }^{11} \mathrm{~B} \mathrm{NMR}\left(128.4 \mathrm{MHz}, \mathrm{CDCl}_{3}\right) \delta$ : -8.2 (br s, 1B); ${ }^{31} \mathrm{P}$ NMR (161 MHz, $\left.\mathrm{CDCl}_{3}\right) \delta$ : 42.8; ESI-HRMS calcd for $\mathrm{C}_{19} \mathrm{H}_{30} \mathrm{BN}_{2} \mathrm{O}_{3} \mathrm{PNa}(\mathrm{M}+\mathrm{Na})^{+}$399.1985, found 399.1981. Meanwhile, (4-dimethylaminopyridine)(2(diethoxyphosphoryl)-2-phenylethyl)dihydroborate (5d') was obtained in $54 \%$ yield ( $92.6 \mathrm{mg}$ as colorless liquid) after column chromatography. ${ }^{1} \mathrm{H}$ NMR (400 MHz, $\left.\mathrm{CDCl}_{3}\right) \delta: 1.19 \sim 1.25(\mathrm{~m}, 6 \mathrm{H}), 1.39 \sim 1.43(\mathrm{~m}, 1 \mathrm{H})$, $2.56 \sim 2.64(\mathrm{~m}, 1 \mathrm{H}), 2.97 \sim 3.05(\mathrm{~m}, 7 \mathrm{H}), 3.88 \sim 4.04(\mathrm{~m}$, $4 \mathrm{H}), 6.39(\mathrm{~d}, J=6.6 \mathrm{~Hz}, 2 \mathrm{H}), 7.09 \sim 7.10(\mathrm{~m}, 1 \mathrm{H}), 7.19 \sim$ $7.20(\mathrm{~m}, 4 \mathrm{H}), 7.97(\mathrm{~d}, J=6.5 \mathrm{~Hz}, 2 \mathrm{H}) ;{ }^{13} \mathrm{C} \mathrm{NMR}(100$ $\left.\mathrm{MHz}, \mathrm{CDCl}_{3}\right) \delta: 16.4(\mathrm{~d}, J=6.5 \mathrm{~Hz}), 16.6(\mathrm{~d}, J=6.1 \mathrm{~Hz})$, $34.3(\mathrm{~d}, J=4.1 \mathrm{~Hz}), 39.4,60.3(\mathrm{~d}, J=4.1 \mathrm{~Hz}), 60.7$ (d, $J=$ $6.7 \mathrm{~Hz}), 105.9,125.0,127.7,129.0,143.8$ (d, $J=14.3 \mathrm{~Hz})$, 147.2, 155.0; ${ }^{11} \mathrm{~B}$ NMR $\left(128.4 \mathrm{MHz}, \mathrm{CDCl}_{3}\right) \delta$ : $-2.5(\mathrm{br}$ $\mathrm{s}, 1 \mathrm{~B}) ;{ }^{31} \mathrm{P} \mathrm{NMR}\left(161 \mathrm{MHz}, \mathrm{CDCl}_{3}\right) \delta$ : 43.2; ESI-HRMS calcd for $\mathrm{C}_{19} \mathrm{H}_{30} \mathrm{BN}_{2} \mathrm{O}_{3} \mathrm{PNa}(\mathrm{M}+\mathrm{Na})^{+}$399.1985, found 399.1981.

\subsection{Radical clock experiment}

A solution of $6(90.2 \mathrm{mg}, 0.417 \mathrm{mmol}), \mathbf{2 a}(68.1 \mathrm{mg}$, $0.500 \mathrm{mmol}), \mathrm{Et}_{3} \mathrm{~B}(209 \mu \mathrm{L}, 0.209 \mathrm{mmol})$, and $\mathrm{PhSH}(21$ $\mu \mathrm{L}, 0.209 \mathrm{mmol})$ in toluene $(2.5 \mathrm{~mL})$ was stirred at room temperature for $9 \mathrm{~h}$ under air. After evaporation of solvent, the resulting crude material was subjected for ${ }^{1} \mathrm{H}$ NMR analysis. The spectra showed that various isomers were obtained, owing to the easy migration of the $\mathrm{C}-\mathrm{C}$ double bond. Despite the complexity of the spectrum, the presence of new vinyl protons and their splitting patterns clearly confirmed the formation of ring opening products. Purification of the crude reaction mixture by flash column chromatography [silica gel, petroleum ether/ethyl acetate $(V: V=3: 1)]$ gave a mixture of two major isomers 7 
(48.5 mg, $0.138 \mathrm{mmol}$ ) in $33 \%$ yield. The characterization data were given for the two isomers mixture. Colorless liquid; $\left.{ }^{1} \mathrm{H} \mathrm{NMR} \mathrm{(400} \mathrm{MHz,} \mathrm{CDCl}_{3}\right) \delta: 0.89(\mathrm{t}, J=7.6 \mathrm{~Hz}$, $3 \mathrm{H} \times 1.84), 1.01(\mathrm{t}, J=7.6 \mathrm{~Hz}, 3 \mathrm{H} \times 1), 1.06 \sim 1.15(\mathrm{~m}$, $3 \mathrm{H} \times 1+3 \mathrm{H} \times 1.84), 1.93(\mathrm{qd}, J=7.6,7.6 \mathrm{~Hz}, 2 \mathrm{H} \times 1.84)$, $2.18 \sim 2.27(\mathrm{~m}, \quad 2 \mathrm{H} \times 1), 2.90 \sim 2.97(\mathrm{~m}, 1 \mathrm{H} \times 1.84), 3.08$ $(\mathrm{s}, 6 \mathrm{H} \times 1), 3.09(\mathrm{~s}, 6 \mathrm{H} \times 1.84), 3.18 \sim 3.25(\mathrm{~m}, 1 \mathrm{H} \times 1)$, $3.89 \sim 4.09(\mathrm{~m}, 2 \mathrm{H} \times 1+2 \mathrm{H} \times 1.84), 5.46(\mathrm{t}, J=7.2 \mathrm{~Hz}$, $1 \mathrm{H} \times 1), 5.60(\mathrm{t}, J=7.2 \mathrm{~Hz}, 1 \mathrm{H} \times 1.84), 6.43(\mathrm{~d}, J=7.2 \mathrm{~Hz}$, $2 \mathrm{H} \times 1), 6.48(\mathrm{~d}, J=7.2 \mathrm{~Hz}, 2 \mathrm{H} \times 1.84), 7.09 \sim 7.41(\mathrm{~m}$, $5 \mathrm{H} \times 1+5 \mathrm{H} \times 1.84), 7.90 \sim 8.05(\mathrm{~m}, 2 \mathrm{H} \times 1+2 \mathrm{H} \times 1.84)$; ${ }^{13} \mathrm{C} \mathrm{NMR}\left(100 \mathrm{MHz}, \mathrm{CDCl}_{3}\right) \delta: 14.4,14.9,22.7,29.7$, $39.4,58.9,59.0,105.8,106.0,125.4,125.5,127.1,127.4$, $127.7,128.3,128.7,131.7,139.2,140.9,144.3,146.5$, $146.8,146.9,154.9,155.0,178.6,178.9 ;{ }^{11} \mathrm{~B}$ NMR $(128.4$ $\left.\mathrm{MHz}, \mathrm{CDCl}_{3}\right) \delta$ : -6.2 (br s, 1B); ESI-HRMS calcd for $\mathrm{C}_{21} \mathrm{H}_{29} \mathrm{BN}_{2} \mathrm{O}_{2} \mathrm{Na}(\mathrm{M}+\mathrm{Na})^{+}$375.2220, found 375.2227.

\subsection{Synthetic applications}

tert-Butyldimethyl(3-(naphthalen-2-yl)-2-(4,4,5,5-tetramethyl-1,3,2-dioxaborolan-2-yl)propoxy)silane (8): To a solution of $3 \mathrm{ka}$ (193 mg, $0.533 \mathrm{mmol})$ in anhydrous THF was slowly added DIBAL-H $(1.10 \mathrm{~mL}, 1.60 \mathrm{mmol})$ at $-78{ }^{\circ} \mathrm{C}$. The reaction mixture was stirred at this temperature for $30 \mathrm{~min}$. The reaction was then allowed to warm to room temperature, a saturated aqueous solution of potassium sodium tartrate (Rochelle salt) was added dropwise and then the mixture was passed through a Celite pad. The mixture was diluted with ethyl acetate and water. The layers were separated and the aqueous layer was extracted with ethyl acetate. The combined organic layers were dried over anhydrous $\mathrm{Na}_{2} \mathrm{SO}_{4}$, filtered, and concentrated under vacuum. To a solution of the crude product, NCS (85.4 $\mathrm{mg}, 0.640 \mathrm{mmol})$, pinacol (126 mg, $1.07 \mathrm{mmol})$, and $\mathrm{NEt}_{3}$ $(148 \mu \mathrm{L}, 1.07 \mathrm{mmol})$ in toluene $(5.0 \mathrm{~mL})$ were added at $0{ }^{\circ} \mathrm{C}$ and then gradually warmed to room temperature. The resulting mixture was stirred at room temperature until thin-layer chromatography (TLC) indicating the reaction complete. The solvent was removed through evaporation. The crude residue was purified by flash column chromatography on silica gel [petroleum ether/ethyl acetate $(V$ : $V=5: 1)]$ to give a pale yellow liquid. To a solution of the pale yellow liquid $(75.1 \mathrm{mg}, 0.405 \mathrm{mmol})$ in anhydrous dichloromethane $(2.0 \mathrm{~mL})$ and tert-butyldimethylsilyl chloride (73.2 mg, $0.486 \mathrm{mmol}$ ), imidazole (41.4 mg, 0.608 mmol) were added at $0{ }^{\circ} \mathrm{C}$ and then gradually warmed to room temperature. The mixture was stirred for $9 \mathrm{~h}$ at this temperature and then concentrated in vacuo. After evaporation under vacuum, the residue was purified by flash column chromatography [silica gel, petroleum ether/ethyl acetate $(V: V=3: 1)]$ to give $8(143 \mathrm{mg}, 0.336 \mathrm{mmol})$ in $63 \%$ yield over three steps. Colorless liquid. ${ }^{1} \mathrm{H}$ NMR (400 $\left.\mathrm{MHz} \mathrm{CDCl}_{3}\right) \delta: 0.08(\mathrm{~s}, 6 \mathrm{H}), 0.97(\mathrm{~s}, 9 \mathrm{H}), 1.20(\mathrm{~d}, J=7.2$ $\mathrm{Hz}, 12 \mathrm{H}), 1.72(\mathrm{q}, J=7.2 \mathrm{~Hz}, 1 \mathrm{H}), 2.92 \sim 3.05(\mathrm{~m}, 2 \mathrm{H})$, $3.69 \sim 3.80(\mathrm{~m}, 2 \mathrm{H}), 7.39 \sim 7.49(\mathrm{~m}, 3 \mathrm{H}), 7.71(\mathrm{~s}, 1 \mathrm{H})$, $7.75 \sim 7.84(\mathrm{~m}, 3 \mathrm{H}) ;{ }^{13} \mathrm{C} \mathrm{NMR}\left(100 \mathrm{MHz}, \mathrm{CDCl}_{3}\right) \delta:-$ 5.4, 18.3, 24.8 (d, $J=6.7 \mathrm{~Hz}), 26.0,33.3,63.4,83.1,124.9$,
125.6, 127.0, 127.4, 127.5 (d, $J=2.1 \mathrm{~Hz}), 127.9$, 131.9, 133.5, 139.6; ${ }^{11} \mathrm{~B}$ NMR (128.4 MHz, $\mathrm{CDCl}_{3}$ ) $\delta: 34.0$ (br s, 1B); ESI-HRMS calcd for $\mathrm{C}_{25} \mathrm{H}_{29} \mathrm{BO}_{3} \mathrm{SiNa}(\mathrm{M}+\mathrm{Na})^{+}$ 449.2659, found 449.2648 .

tert-Butyl(2-(furan-2-yl)-3-(naphthalen-2-yl)propoxy)dimethylsilane (9): A solution of furan $(55 \mu \mathrm{L}, 0.760 \mathrm{mmol})$ in THF $(6.0 \mathrm{~mL})$ was cooled to $-78{ }^{\circ} \mathrm{C}$ and treated with $n$-BuLi $(0.48 \mathrm{~mL}, 1.6 \mathrm{~mol} / \mathrm{L}$ in hexanes $)$. The cooling bath was removed and the mixture was stirred at room temperature for $1 \mathrm{~h}$. The mixture was cooled to $-78{ }^{\circ} \mathrm{C}$ and a solution of boronic ester $8(216 \mathrm{mg}, 0.507 \mathrm{mmol})$ in THF $(1.5 \mathrm{~mL})$ was added by dropwise. The mixture was stirred at $-78{ }^{\circ} \mathrm{C}$ for overnight. A solution of $N$-bromo-succinimide (NBS) (136 mg, $0.764 \mathrm{mmol})$ in THF $(1.5 \mathrm{~mL})$ was added by dropwise. After $1 \mathrm{~h}$ at $-78{ }^{\circ} \mathrm{C}$, the reaction was quenched with saturated $\mathrm{Na}_{2} \mathrm{~S}_{2} \mathrm{O}_{3}$ aqueous solution, and the reaction mixture was allowed to warm to room temperature. The reaction mixture was diluted with ethyl acetate and water. The layers were separated and the aqueous layer was extracted with ethyl acetate. The combined organic layers were dried over anhydrous $\mathrm{Na}_{2} \mathrm{SO}_{4}$, filtered, and concentrated under vacuum. The crude residue was purified by flash column chromatography on silica gel [petroleum ether/ethyl acetate $(V: V=3: 1)$ ] to give 9 (139.3 mg, $0.380 \mathrm{mmol}$ ) in $75 \%$ yield. Yellow liquid. ${ }^{1} \mathrm{H}$ NMR $\left(400 \mathrm{MHz}, \mathrm{CDCl}_{3}\right) \delta$ : $-0.02(\mathrm{~d}, J=2.6 \mathrm{~Hz}, 6 \mathrm{H})$, $0.89 \sim 0.90(\mathrm{~m}, 9 \mathrm{H}), 3.05 \sim 3.12(\mathrm{~m}, 1 \mathrm{H}), 3.19 \sim 3.28(\mathrm{~m}$, $2 \mathrm{H}), 3.74 \sim 3.78(\mathrm{~m}, 2 \mathrm{H}), 5.97(\mathrm{dd}, J=3.2,7.3 \mathrm{~Hz}, 1 \mathrm{H})$, $6.14 \sim 6.25(\mathrm{~m}, 1 \mathrm{H}), 7.22(\mathrm{dd}, J=1.6,8.5 \mathrm{~Hz}, 1 \mathrm{H}), 7.32$ $(\mathrm{dd}, J=0.8,1.8 \mathrm{~Hz}, 1 \mathrm{H}), 7.40 \sim 7.45(\mathrm{~m}, 2 \mathrm{H}), 7.54 \sim 7.56$ $(\mathrm{m}, 1 \mathrm{H}), 7.70 \sim 7.80(\mathrm{~m}, 3 \mathrm{H}) ;{ }^{13} \mathrm{C} \mathrm{NMR}(100 \mathrm{MHz}$, $\left.\mathrm{CDCl}_{3}\right) \delta:-5.6(\mathrm{~d}, J=9.3 \mathrm{~Hz}), 18.3,25.9,36.2,43,7$, 64.1, 106.3, 110.0, 125.1, 125.7, 127.4 (d, $J=2.3 \mathrm{~Hz})$, $127.6(\mathrm{~d}, J=2.3 \mathrm{~Hz}), 127.7,132.0,133.4,137.5,140.7$, 155.9; ESI-HRMS calcd for $\mathrm{C}_{23} \mathrm{H}_{31} \mathrm{O}_{2} \mathrm{Si}(\mathrm{M}+\mathrm{H})$ 367.2093, found 367.2094.

4-(1-((tert-Butyldimethylsilyl)oxy)-3-(naphthalen-2-yl)propan-2-yl)-3-fluoropyridine (10): To a solution of $(i \text {-Pr })_{2} \mathrm{NH}(76 \mu \mathrm{L}, 0.539 \mathrm{mmol})$ in THF $(1.0 \mathrm{~mL})$ cooled to $-78{ }^{\circ} \mathrm{C}$ was add $n$-BuLi $(0.24 \mathrm{~mL}, 1.6 \mathrm{~mol} / \mathrm{L}$ in hexane, $0.600 \mathrm{mmol}$ ), and then the mixture was stirred at $-60{ }^{\circ} \mathrm{C}$ for $1 \mathrm{~h}$. A solution of 3-fluoropyridine $(58 \mu \mathrm{L}, 0.675$ $\mathrm{mmol})$ in THF $(0.5 \mathrm{~mL})$ was added by dropwise and the mixture was stirred at $-60{ }^{\circ} \mathrm{C}$ for $1 \mathrm{~h}$. A solution of 8 $(115 \mathrm{mg}, 0.270 \mathrm{mmol})$ in THF $(1.0 \mathrm{~mL})$ was added and the mixture was stirred for $12 \mathrm{~h}$ at $-60{ }^{\circ} \mathrm{C}$. Troc-Cl $(149 \mu \mathrm{L}$, $1.082 \mathrm{mmol}$ ) was added, and the mixture was stirred at $60{ }^{\circ} \mathrm{C}$ for $5 \mathrm{~h}$ and then gradually warmed to room temperature. The mixture was diluted with $\mathrm{Et}_{2} \mathrm{O}$ and $\mathrm{H}_{2} \mathrm{O}$, the layers were separated and the aqueous phase was neutralized with saturated aqueous $\mathrm{NaHCO}_{3}$ solution. The aqueous layer was extracted with $\mathrm{Et}_{2} \mathrm{O}$, the combined organic layers were dried over $\mathrm{MgSO}_{4}$, filtered and evaporated. The residue was dissolved in THF $(2.0 \mathrm{~mL})$ under air atmosphere at room temperature for $3 \mathrm{~h}$. After that, the reaction mixture was diluted with water, and the aqueous phase was extracted with $\mathrm{Et}_{2} \mathrm{O}$. The organic layers were washed 
with brine, dried over $\mathrm{MgSO}_{4}$, filtered, and evaporated. Purification by flash chromatography on silica gel [petroleum ether/ethyl acetate $(V: V=3: 1)]$ to give $\mathbf{1 0}(64.7$ $\mathrm{mg}, 0.164 \mathrm{mmol})$ in $60 \%$ yield. Colorless liquid. ${ }^{1} \mathrm{H}$ NMR $\left(400 \mathrm{MHz}, \mathrm{CDCl}_{3}\right) \delta:-0.04(\mathrm{~s}, 6 \mathrm{H}), 0.88(\mathrm{~s}, 9 \mathrm{H}), 3.07$ $(\mathrm{dd}, J=7.8,13.6 \mathrm{~Hz}, 1 \mathrm{H}), 3.34$ (dd, $J=7.5,13.7 \mathrm{~Hz}, 1 \mathrm{H})$, $3.53 \sim 3.60(\mathrm{~m}, 1 \mathrm{H}), 3.80(\mathrm{~d}, J=5.2 \mathrm{~Hz}, 2 \mathrm{H}), 7.27 \sim 7.30$ $(\mathrm{m}, 2 \mathrm{H}), 7.42 \sim 7.45(\mathrm{~m}, 2 \mathrm{H}), 7.57(\mathrm{~s}, 1 \mathrm{H}), 7.73 \sim 7.81(\mathrm{~m}$, $3 \mathrm{H}), 8.30 \sim 8.37(\mathrm{~m}, 2 \mathrm{H}) ;{ }^{13} \mathrm{C}$ NMR $\left(100 \mathrm{MHz}, \mathrm{CDCl}_{3}\right) \delta$ : $-5.7(\mathrm{~d}, J=3.6 \mathrm{~Hz}), 18.1,25.7,36.4,42.3,64.2,124.2$, $125.4,126.0,127.3,127.4(\mathrm{~d}, J=2.9 \mathrm{~Hz}), 127.6,128.0$, 132.1, 133.4, 136.6, 137.8 (d, $J=25.7 \mathrm{~Hz}), 138.5$ (d, $J=$ $12.4 \mathrm{~Hz}), 145.3$ (d, $J=4.9 \mathrm{~Hz}), 157.2,159.2 ;{ }^{19} \mathrm{~F}$ NMR $\left(376 \mathrm{MHz} \mathrm{CDCl}_{3}\right) \delta$ : -132.41 (s, 1F); ESI-HRMS calcd for $\mathrm{C}_{24} \mathrm{H}_{31} \mathrm{FNOSi}(\mathrm{M}+\mathrm{H})^{+}$396.2159, found 396.2158.

3-((tert-Butyldimethylsilyl)oxy)-2-(naphthalen-2-ylmethyl)propan-1-ol (11): To the solution of 8 (115 mg, 0.270 $\mathrm{mmol})$ in dry THF $(2.5 \mathrm{~mL})$ was added bromochloromethane $(44 \mu \mathrm{L}, 0.677 \mathrm{mmol})$ under nitrogen. The resulting solution was cooled to $-78{ }^{\circ} \mathrm{C}$ and treated with $n$-BuLi (1.6 mol/L in hexane) $(162 \mu \mathrm{L}, 0.405 \mathrm{mmol})$ dropwise. After stirring at $-78{ }^{\circ} \mathrm{C}$ for $20 \mathrm{~min}$, the reaction mixture was allowed to warm to room temperature slowly and stirred for additional $18 \mathrm{~h}$. The resulting mixture was cooled to $0{ }^{\circ} \mathrm{C}$ and water $(2.0 \mathrm{~mL})$ was added slowly. The mixture was extracted with diethyl ether for three times. The combined organic layers were washed with brine, dried over $\mathrm{Na}_{2} \mathrm{SO}_{4}$, filtered, and concentrated under reduced pressure. The crude product was dissolved in $1.5 \mathrm{~mL}$ of methanol/MeCN $(V: V=1: 1)$ before addition of $\mathrm{H}_{2} \mathrm{O}_{2}(30 \%$ aqueous solution, $247 \mu \mathrm{L}, 2.16 \mathrm{mmol})$. The reaction mixture was stirred at $40{ }^{\circ} \mathrm{C}$ for $20 \mathrm{~h}$. The reaction mixture was diluted with ethyl acetate and water. The layers were separated and the aqueous layer was extracted with ethyl acetate. The combined organic layers were dried anhydrous $\mathrm{Na}_{2} \mathrm{SO}_{4}$, filtered, and concentrated under vacuum. The crude residue was purified by flash chromatography to afford product 11 (39.7 mg, 0.183 $\mathrm{mmol})$ in $68 \%$ yield. Colorless liquid. ${ }^{1} \mathrm{H}$ NMR $(400 \mathrm{MHz}$, $\left.\mathrm{CDCl}_{3}\right) \delta: 0.08(\mathrm{~d}, J=4.4 \mathrm{~Hz}, 6 \mathrm{H}), 0.94(\mathrm{~s}, 9 \mathrm{H}), 2.10 \sim$ $2.15(\mathrm{~m}, 1 \mathrm{H}), 2.81 \sim 2.85(\mathrm{~m}, 2 \mathrm{H}), 3.66 \sim 3.76(\mathrm{~m}, 2 \mathrm{H})$, $3.79 \sim 3.83(\mathrm{~m}, 2 \mathrm{H}), 7.35(\mathrm{dd}, J=1.6,6.8 \mathrm{~Hz}, 1 \mathrm{H}), 7.43 \sim$ $7.49(\mathrm{~m}, 2 \mathrm{H}), 7.65(\mathrm{~s}, 1 \mathrm{H}), 7.78 \sim 7.83(\mathrm{~m}, 3 \mathrm{H}) ;{ }^{13} \mathrm{C} \mathrm{NMR}$ $\left(100 \mathrm{MHz}, \mathrm{CDCl}_{3}\right) \delta:-5.6,18.1,25.9,29.7,34.3,43.8$, $65.6,66.0,125.2,125.9,127.3,127.4,127.6,127.9,132.1$, 133.5, 137.6; ESI-HRMS calcd for $\mathrm{C}_{14} \mathrm{H}_{14} \mathrm{SiONa}(\mathrm{M}+$ $\mathrm{Na})^{+}$353.1913, found 353.1909.

Supporting Information The NMR spectra of reported new compounds is available free of charge via the Internet at http://sioc-journal.cn/.

\section{References}

[1] Hall, D. G. Boronic Acids: Preparation and Applications in Organic Synthesis, Medicine and Materials, 2nd ed., Wiley-VCH, Weinheim, Germany, 2011.

[2] (a) Lei, Z.; Huang, Z. Synlett 2013, 24, 1745. (b) Beletskaya, I.; Pelter, A. Tetrahedron 1997, 53, 4957.

(c) Burgess, K.; Ohlmeyer, M. J. Chem. Rev. 1991, 91, 1179.

(d) Chen, J.-B.; Whiting, A. Synthesis 2018, 50, 3843.

[3] (a) Schiffner, J. A.; Müther, K.; Oestreich, M. Angew. Chem., Int. Ed. 2010, 49, 1194.

(b) Mantilli, L.; Mazet, C. ChemCatChem 2010, 2, 501.

(c) Calow, A. D. J.; Whiting, A. Org. Biomol. Chem. 2012, 10, 5485 .

(d) Stavber, G.; Časar, Z. ChemCatChem 2014, 6, 2162.

(e) Lee, S.; Yun, J. In Synthesis and Application of Organoboron Compounds, Vol. 49, Eds.: Fernandez, E.; Whiting, A., 2015, pp. $73 \sim 92$.

(f) Collins, B. S. L.; Wilson, C. M.; Myers, E. L.; Aggarwal, V. K. Angew. Chem., Int. Ed. 2017, 56, 11700.

(g) Liu, Y.; Zhang, W. Chin. J. Org. Chem. 2016, 36, 2249 (in Chinese).

(刘媛媛, 张万斌, 有机化学, 2016, 36, 2249.)

[4] Ibrahim, M. R.; Bühl, M.; Knab, R.; Schleyer, P. V. R. J. Comput. Chem. 1992, 13, 423.

[5] (a) He, Z.; Zajdlik, A.; Yudin, A. K. Acc. Chem. Res. 2014, 47, 1029 .

(b) He, Z.; Zajdlik, A.; Yudin, A. K. Dalton Trans. 2014, 43, 11434.

[6] Sucrow, W.; Zühlke, L.; Slopianka, M.; Pickardt, J. Chem. Ber. 1977, 110, 2818.

[7] Ng, E. W. H.; Low, K.-H.; Chiu, P. J. Am. Chem. Soc. 2018, 140, 3537.

[8] Radcliffe, J. E.; Fasano, V.; Adams, R. W.; You, P.; Ingleson, M. J. Chem. Sci. 2019, 10, 1434

[9] (a) Jin, J.; Xia, H.; Zhang, F.; Wang, Y. Chin. J. Org. Chem. 2020, 40, 2185 (in Chinese).

(靳继康, 夏慧敏, 张凤莲, 汪义丰, 有机化学, 2020, 40, 2185.)

(b) Zhang, F.-L.; Wang, Y.-F. In Science of Synthesis: Advances in Organoboron Chemistry towards Organic Synthesis, Ed.: Fernández, E., Thieme, Stuttgart, 2019, pp. 355 392.

(c) Taniguchi, T. Eur. J. Org. Chem. 2019, 2019, 6308.

(d) Yang, J.; Li, Z.; Zhu, S. Chin. J. Org. Chem. 2017, 37, 2481 (in Chinese).

(杨吉民, 李子奇, 朱守非, 有机化学, 2017, 37, 2481.)

(e) Xu, A.-Q.; Zhang, F.-L.; Ye, T.; Yu, Z.-X.; Wang, Y.-F. CCS Chem. 2019, 1, 504.

(f) Ueng, S.-H.; Makhlouf Brahmi, M.; Derat, É.; Fensterbank, L.; Lacôte, E.; Malacria, M.; Curran, D. P. J. Am. Chem. Soc. 2008 130, 10082.

[10] (a) Ren, S.-C.; Zhang, F.-L.; Qi, J.; Huang, Y.-S.; Xu, A.-Q.; Yan, H.-Y.; Wang, Y.-F. J. Am. Chem. Soc. 2017, 139, 6050.

(b) Watanabe, T.; Hirose, D.; Curran, D. P.; Taniguchi, T. Chem. Eur. J. 2017, 23, 5404.

(c) Zhou, N.; Yuan, X.-A.; Zhao, Y.; Xie, J.; Zhu, C. Angew. Chem., Int. Ed. 2018, 57, 3990.

(d) Dai, W.; Geib, S. J.; Curran, D. P. J. Am. Chem. Soc. 2019, 141, 12355.

(e) Qi, J.; Zhang, F.-L.; Jin, J.-K.; Zhao, Q.; Li, B.; Liu, L.-X.; Wang, Y.-F. Angew. Chem., Int. Ed. 2020, 59, 12876.

(f) Xia, P. J.; Song, D.; Ye, Z. P.; Hu, Y. Z.; Xiao, J. A.; Xiang, H. Y.; Chen, X. Q.; Yang, H. Angew. Chem., Int. Ed. 2020, 59, 6706.

(g) Zhu, C.; Dong, J.; Liu, X.; Gao, L.; Zhao, Y.; Xie, J.; Li, S.; Zhu, C. Angew. Chem., Int. Ed. 2020, 59, 12817.

(h) Xu, W.; Jiang, H.; Leng, J.; Ong, H. W.; Wu, J. Angew. Chem., Int. Ed. 2020, 59, 4009.

(i) Dai, W.; Geib, S. J.; Curran, D. P. J. Am. Chem. Soc. 2020, 142, 6261 .

(j) Kawamoto, T.; Morioka, T.; Noguchi, K.; Curran, D. P.; Kamimura, A. Org. Lett. 2021, 23, 1825.

(k) Liu, Y.; Li, J.-L.; Liu, X.-G.; Wu, J.-Q.; Huang, Z.-S.; Li, Q.; Wang, H. Org. Lett. 2021, 23, 1891.

(1) Xu, H.; Jiang, Z. Chin. J. Org. Chem. 2020, 40, 3483 (in Chinese).

(许荷欢, 江智勇, 有机化学, 2020, 40, 3483.) 
[11] (a) Ren, S.-C.; Zhang, F.-L.; Xu, A.-Q.; Yang, Y.; Zheng, M.; Zhou, X.; Fu, Y.; Wang, Y.-F. Nat. Commun. 2019, 10, 1934.

(b) Huang, Y.-S.; Wang, J.; Zheng, W.-X.; Zhang, F.-L.; Yu, Y.-J.; Zheng, M.; Zhou, X.; Wang, Y.-F. Chem. Commun. 2019, 55, 11904.

[12] Brahmi, M. M.; Monot, J.; Desage-El Murr, M.; Curran, D. P.; Fensterbank, L.; Lacôte, E.; Malacria, M. J. Org. Chem. 2010, 75, 6983.

[13] (a) Lalevée, J.; Blanchard, N.; Tehfe, M.-A.; Chany, A.-C.; Fouassier, J.-P. Chem.-Eur. J. 2010, 16, 12920.

(b) Lalevée, J.; Blanchard, N.; Chany, A.-C.; Tehfe, M.-A.; Allonas, X.; Fouassier, J.-P. J. Phys. Org. Chem. 2009, 22, 986.

(c) Jin, J.-K.; Zhang, F.-L.; Wang, Y.-F. Acta Chim. Sinica 2019, 77, 889 (in Chinese).

(靳继康, 张凤莲, 汪义丰, 化学学报, 2019, 77, 889.)
[14] Yu, Y.-J.; Zhang, F.-L.; Peng, T.-Y.; Wang, C.-L.; Cheng, J.; Chen, C.; Houk, K. N.; Wang, Y.-F. Science 2021, 371, 1232.

[15] (a) Roberts, B. P. Chem. Soc. Rev. 1999, 28, 25. (b) Pan, X.; Lacôte, E.; Lalevée, J.; Curran, D. P. J. Am. Chem. Soc. 2012, 134, 5669.

[16] Staubitz, A.; Robertson, A. P. M.; Sloan, M. E.; Manners, I. Chem. Rev. 2010, 110, 4023.

[17] (a) Griller, D.; Ingold, K. U. Acc. Chem. Res. 1980, 13, 317.

(b) Newcomb, M. Tetrahedron 1993, 49, 1151.

[18] Dénès, F.; Pichowicz, M.; Povie, G.; Renaud, P. Chem. Rev. 2014 $114,2587$.

[19] (a) Bonet, A.; Odachowski, M.; Leonori, D.; Essafi, S.; Aggarwal, V. K. Nat. Chem. 2014, 6, 584.

(b) Llaveria, J.; Leonori, D.; Aggarwal, V. K. J. Am. Chem. Soc. 2015, 137, 10958.

(Cheng, F.) 